\title{
Variabilité spatio-temporelle de la biomasse microphytobenthique en rade de Brest et en Manche occidentale
}

\author{
Gaëlle SAGAN, Gérard THOUZEAU*
}

UMR 6539 CNRS, université de Bretagne occidentale, Institut universitaire européen de la mer, Technopôle Brest-Iroise, place N.-Copernic, 29280 Plouzané, France

(Reçu le 01/04/97, révisé le 11/07/98, accepté le 18/07/98)

\begin{abstract}
Microphytobenthic biomass in the Bay of Brest and the western English Channel. Microphytobenthic biomass was estimated in the Bay of Brest (1994) and the western English Channel (Trezen Vraz, 1993) using spectrophotometry. Best results ( $12 \%$ difference) were obtained with an instantaneous extraction procedure at room temperature, compared with the cold extraction procedure. Chlorophyll biomass in sediment was higher in the western English Channel (65-215 $\mathrm{mg} \mathrm{Chl} a \mathrm{~m}^{-2}$ ) than in the Bay of Brest (10-113 $\mathrm{mg} \mathrm{Chl} a \mathrm{~m}^{-2}$ ), in contrast to total pigment biomass (Chl $a+$ pheo $a: 88-254 \mathrm{mg} \mathrm{m}^{-2}$ at Trezen Vraz vs. $131-934 \mathrm{mg} \mathrm{m}^{-2}$ in the Bay of Brest). This study emphasized decreasing pigment biomass from the estuary to the open sea. Low benthic pigment biomass in the Bay, compared to eutrophic ecosystems, agrees with previous studies on the pelagic system, suggesting that there is no eutrophication in the Bay of Brest. Microphytobenthic blooms were strongly correlated with environmental factors, such as river floods, light intensity, water temperature and the spring/neap tidal cycle. Turbidity and light could be limiting factors at Roscanvel, i.e. the deepest site facing gyre circulation. The latter would favour sedimentation of silt particles at the sediment-water interface. Microphytobenthos population dynamics would be also dependent on the spring/neap tidal cycle, since maximum chlorophyll biomass occurred at neap tides at all three sites in the Bay of Brest, in contrast to the western English Channel. Lower temporal variations of pigment biomass in sediment were observed in the Channel, compared with the Bay of Brest; maximum chlorophyll biomass in sediment was found to occur three to four weeks after the phytoplankton blooms. (O) Elsevier, Paris
\end{abstract} microphytobenthos / biomass / coastal ecosystems / physical forcing

Résumé - La biomasse microphytobenthique a été estimée par spectrophotométrie en rade de Brest (1994) et en Manche occidentale (1993). La comparaison de deux protocoles d'extraction pigmentaire montre que l'extraction instantanée à température ambiante est supérieure de $42 \%$, à l'extraction conventionnelle à froid. La biomasse chlorophyllienne en Manche (65-215 $\mathrm{mg} \mathrm{ChI} a \mathrm{~m}^{-2}$ ) est supérieure à celle de la rade de Brest (10-113 $\mathrm{mg} \mathrm{Chl} a \mathrm{~m}^{-2}$ ), mais la biomasse pigmentaire totale (Chl $a+$ phéo $a$ ) est deux à quatre fois plus élevée dans les sédiments de la rade (131-934 $\mathrm{mg} \mathrm{m}^{-2}$ ) que dans les sédiments du Trezen Vraz $\left(88-254 \mathrm{mg} \mathrm{m}^{-2}\right)$. Un gradient de biomasse décroissant de l'estuaire vers la mer ouverte est observé. Les biomasses pigmentaires en rade de Brest sont inférieures à celles observées dans les systèmes marins eutrophisés, confortant ainsi plusieurs études du système pélagique selon lesquelles la rade de Brest constitue un milieu eutrophe mais non eutrophisé. Les floraisons microphytobenthiques en rade de Brest sont fortement dépendantes de l'évolution des facteurs physico-chimiques du milieu (crues des rivières, lumière, température, cycles de marée). En particulier, l'impact des variations de l'intensité du mélange turbulent (et de la lumière) est montré lors de l'alternance vive-eau/morteeau, les maxima de biomasse chlorophyllienne dans le sédiment étant observés en périodes de morte-eau. En Manche en revanche, les variations temporelles de la biomasse pigmentaire dans les sédiments sont de faible amplitude, avec des pics de biomasse en vive-eau ; la biomasse pigmentaire dans le sédiment augmente en moyenne trois à quatre semaines après les blooms planctoniques. (C) Elsevier, Paris

microphytobenthos / biomasse / écosystèmes côtiers / forçage physique

* Correspondance et tirés-à-part 


\section{INTRODUCTION}

Le microphytobenthos constitue un maillon trop souvent négligé dans les réseaux trophiques benthiques des écosystèmes côtiers peu profonds. Dans le cadre de la modélisation du cycle du carbone dans ces réseaux, la détermination de la biomasse et de la production microphytobenthiques est essentielle pour estimer les flux trophiques liés à la microflore. Ce travail s'inscrit dans le cadre du programme Rade de Brest (contrat de Baie) et du programme national d'océanographie côtière (PNOC). Il concerne l'estimation de la biomasse microphytobenthique sur les zones de captage de naissain de coquille Saint-Jacques en rade de Brest et sur le site atelier $\mathrm{n}^{\circ} 1 \mathrm{du}$ chantier Manche du PNOC (Trezen Vraz).

L'importance quantitative des microphytes benthiques marins et leur rôle fonctionnel dans les réseaux trophiques côtiers ont longtemps été sous-estimés [53], bien que le microphytobenthos colonise toutes les strates intertidales et subtidales [58]. La concentration en pigments photosynthétiques du sédiment, généralement la chlorophylle $a(\mathrm{Chl} a)$, est souvent utilisée comme indicateur de la variabilité spatiale et temporelle de la biomasse microphytobenthique $[54,66]$, malgré (1) l'origine diverse des pigments dans le sédiment (microphytobenthos per se, phytoplancton sédimenté, débris de macrophytes, matériel particulaire végétal issu des rives et des bassins versants et pelotes fécales [51], (2) la variabilité du rapport $\mathrm{C} / \mathrm{Chl} a$ chez les diatomées benthiques [16], et (3) une corrélation parfois faible entre le nombre de cellules et la concentration en pigments [63]. Cependant, plusieurs études ont montré que la production primaire microphytobenthique à la surface des sédiments pouvait être estimée à partir des concentrations en $\mathrm{Chl} a[7,12$, $15,46]$. D'autre part, les produits de dégradation (phéophorbide $a$-like surtout et autres phéophorbides [59]) constituent des indicateurs de l'état physiologique, du contenu détritique et de l'intensité des processus de broutage au sein des populations de microphytes [38, 40, 53].

Les mesures de concentration en pigments du microphytobenthos font le plus souvent appel aux techniques développées pour le phytoplancton. Hormis les techniques récentes de chromatographie (HPLC), la Chl $a$ et d'autres pigments sont habituellement dosés, après extraction dans un solvant organique, par fluorimétrie $[24,28,35$, $39,61,70]$ ou spectrophotométrie $[36,44,56]$.

Cette étude a pour objectifs de caractériser la dynamique spatio-temporelle du microphytobenthos dans les sédiments de deux écosystèmes côtiers macrotidaux, la rade de Brest et le Trezen Vraz (Manche occidentale), et de quantifier la place occupée par les populations microphytobenthiques dans les réseaux trophiques de ces écosystèmes. L'intégration de ces résultats dans les modèles numériques ponctuels à l'état stable et les modèles de simulation dynamique des réseaux trophiques pélagobenthiques (coll. Ifremer), contribuera à la modélisation du cycle du carbone dans ces écosystèmes côtiers. Les mesures pigmentaires, réalisées par spectrophotométrie, ont nécessité dans un premier temps la mise au point d'un protocole d'extraction des pigments photosynthétiques, Chl $a$ et phéopigments $a$ (phéo $a$ ).

\section{MATÉRIELS ET MÉTHODES}

\subsection{Zones d'étude}

Trois sites ateliers ont été expérimentés entre avril et octobre 1994 en rade de Brest, sur la base d'un suivi temporel mensuel à hebdomadaire (période des blooms printaniers) : Sainte-Anne (SA ; $15 \mathrm{~m}$ de profondeur), Ducs d'Albe (DA ; $20 \mathrm{~m}$ ) et Roscanvel (RO, $30 \mathrm{~m}$; figure I). En 1994, la température de l'eau sur la verticale a été homogène toute l'année à $\mathrm{SA}\left(\Delta \mathrm{T} \leq 0,16^{\circ} \mathrm{C}\right)$; pour les deux autres sites, un gradient vertical a été observé entre la mi-mai et début septembre (de 0,4 à $0,8^{\circ} \mathrm{C}$ à $\mathrm{RO}$ et de 0,2 à $1,48^{\circ} \mathrm{C}$ à $\mathrm{DA}$; [10]). Les mesures de salinité des eaux de la rade confirment le caractère marin des eaux de SA (de 33,95 à 35,15 entre avril et septembre), et le caractère estuarien des eaux de DA $(\Delta S=3,11$ en avril, entre le fond et la surface). La salinité minimale des eaux de fond (SA : 33,95; RO : 33,51; DA : 32,88) souligne la position intermédiaire de $\mathrm{RO}$, par ses caractéristiques physico-chimiques. Cette station est également soumise à des dessalures de surface, de durée inférieure à celle observée à DA. En rade, la dynamique saisonnière des sels nutritifs est essentiellement contrôlée par les apports d'eau douce, notamment en hiver et au printemps, et par l'absorption du phytoplancton durant la période productive. La croissance phytoplanctonique est surtout limitée par les silicates durant les premiers stades de la floraison printanière [49]. L'importance du matériel détritique provenant des apports fluviaux est illustrée par les valeurs du rapport carbone organique particulaire/azote organique particulaire, COP/NOP, du compartiment pélagique total (de 6,6 à 20,4 à RO en 1993 ; [18]). Si l'évolution temporelle de la production totale ne permet pas de mettre en 


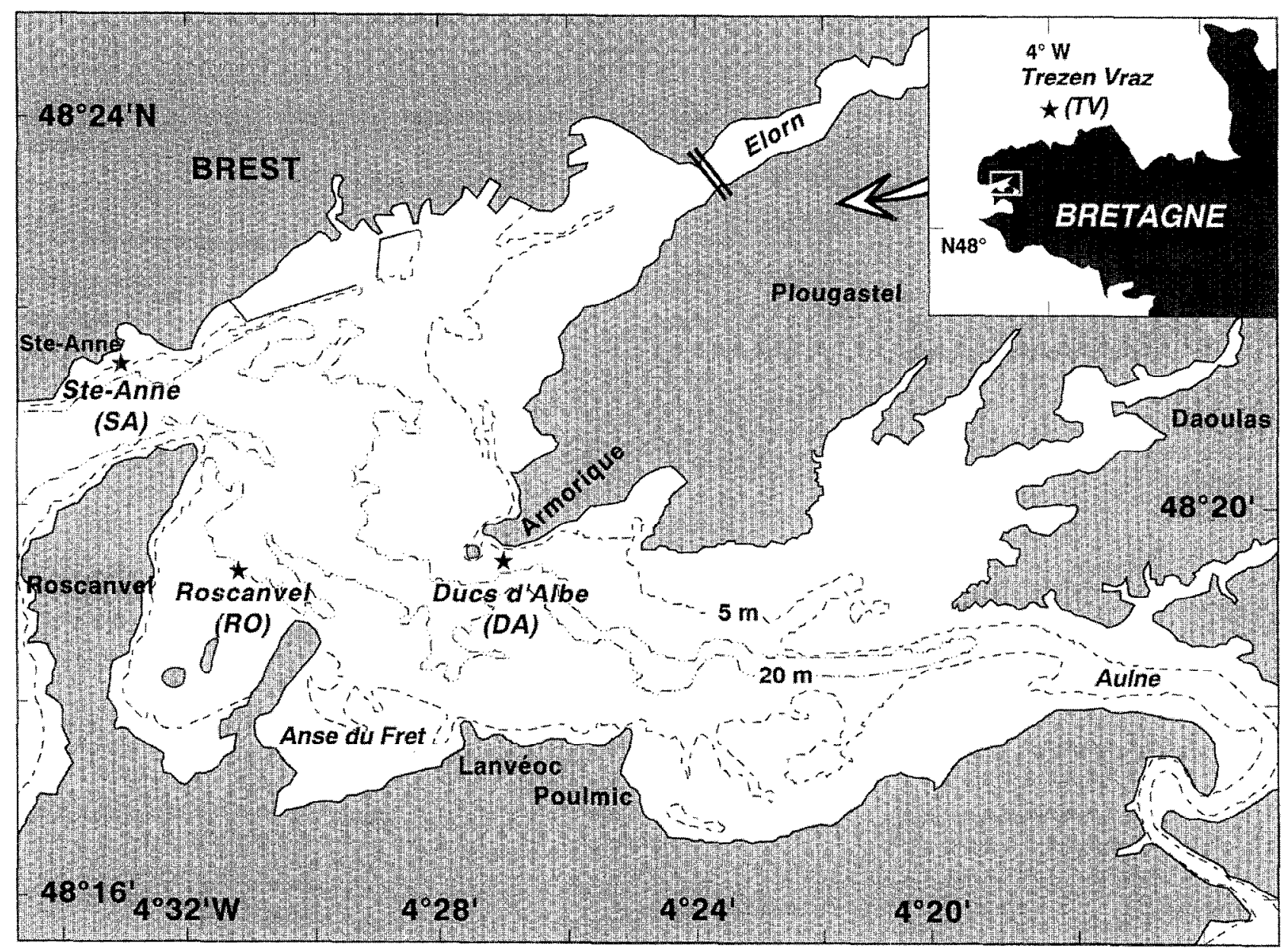

Figure 1. Localisation des stations (*) échantillonnées en rade de Brest en 1994 (Sainte-Anne, Roscanvel et Ducs d'Albe) et en Manche occidentale en 1993 (Trezen Vraz).

Figure 1. Location of study sites $(*)$ sampled in the Bay of Brest in 1994 (Sainte-Anne, Roscanvel and Ducs d'Albe) and in the western English Channel in 1993 (Trezen Vraz).

évidence une augmentation de la productivité de l'écosystème [33], la multiplication estivale des blooms de dinoflagellés toxiques constitue un premier indice de déséquilibre. En ce qui concerne nos sites d'études, les concentrations en Chl $a$ des eaux de fond étaient maximales à DA en 1994 (10 à $14 \mu \mathrm{g} \mathrm{L}{ }^{-1}$ en juin [10 ]).

La granulométrie des sédiments est très variable d'un site à l'autre, DA présentant les sédiments les plus grossiers (médiane : 1005 à $1252 \mu \mathrm{m}$ ) et SA les sédiments les plus fins (médiane : 411 à $623 \mu \mathrm{m}$ [10]). La fraction grossière est dominante dans les sédiments des trois sites (35 à $70 \%$ du poids sec de sédiment), alors que la teneur en pélites varie entre 0,02 et $1,61 \%$ (SA : 0,02 à $0,52 \%$; DA : 0,14 à $1,03 \%$; RO : 0,07 à $1,61 \%)$. La teneur en matière organique $(\mathrm{MO})$ du sédiment fluctue entre 1,6 et $5 \%$, les minima étant observés à SA (1,6 à $2,3 \%$ ), alors que les taux sont comparables à RO $(3,3$ à $4,2 \%)$ et DA (3,9 à $5 \%$; [10]).

Le site atelier $n^{\circ} 1$ du PNOC Manche, ou Trezen Vraz (TV, $48^{\circ} 51^{\prime} 00 \mathrm{~N}, 3^{\circ} 54^{\prime} 00 \mathrm{~W}$; profondeur $71 \mathrm{~m}$ ), est situé au large de Roscoff (figure 1) en zone thermiquement homogène. Il a été choisi en raison du caractère représentatif, à méso-échelle, de son peuplement de macrofaune benthique. La zone atclicr apparticnt à la communauté des sédiments grossiers sablo-graveleux à Venus fasciata, qui s'étend sur les sédiments grossiers propres de la Manche occidentale à une profondeur en général supérieure à 65-70 m. Deux stratégies d'échan- 
tillonnage ont été utilisées dans le cadre du PNOC Manche en 1993 : un échantillonnage mensuel ou bimestriel (N.O. Pluteus; neuf campagnes de février à novembre 1993) et un suivi annuel dans le cadre de CPBMANCHE1 (N.O. Suroit ; 17 mai-15 juin 1993). L'environnement physique est caractérisé par une absence de stratification thermique durable de la colonne d'eau, en été. La température de l'eau en 1993 a varié de $10^{\circ} \mathrm{C}$ en février à $15,8^{\circ} \mathrm{C}$ en août. La zone d'études est caractérisée par des sédiments grossiers propres (selon la classification de Chassé et Glémarec [9]) : graviers (fraction $[2000 ; 10000 \mu \mathrm{m}]=46 \%$ du poids sec de sédiment) et sables grossiers (fraction $[500 ; 2000 \mu \mathrm{m}]=$ $26 \%$ du poids sec). La teneur en pélites (fraction $<63 \mu \mathrm{m}$ ) est toujours $<0,5 \%$ [68]. Le pourcentage de MO dans le sédiment varie de $2,3 \%$ (mars) à $3,6 \%$ (juin) ; les maxima sont observés en février et début juin.

\section{2. Échantillonnage}

Deux stratégies d'échantillonnage ont été appliquées suivant les zones d'étude en raison de contraintes logistiques liées à la profondeur des fonds. Fn Manche, les échantillons de sédiment ont été prélevés à l'aide d'un carottier Reineck modifié, prélevant une surface de $50 \mathrm{~cm}^{2}$. Les profils verticaux n'ayant pu être étudiés sur ces échantillons, une mesure globale $(0-10 \mathrm{~cm})$ du contenu pigmentaire en Chl $a$ et en phéo $a$ a été effectuée. En rade de Brest, les échantillons de sédiment ont été prélevés en plongéc à l'aide de carottes en PVC de diamètre interne $6,8 \mathrm{~cm}$. Pour l'étude des profils verticaux, les carottes ont été fractionnées par «tranches» de 5 mm [54] jusqu'à $3 \mathrm{~cm}$ de profondeur. Les concentrations en $\mathrm{Chl} a$ et phéo $a$ ont également été mesurées dans la couche d'eau surnageante des carottes.

Tous les échantillons ont été congelés à $-20^{\circ} \mathrm{C}$ avant analyse de leur contenu en pigments chlorophylliens. La lyophilisation des échantillons n'a pas été retenue dans la mesure où elle provoquerait une réduction de la concentration en pigments $[32,53,54]$.

\subsection{Test de validité de l'échantillonnage}

Chaque échantillon de sédiment (carottages Reineck et plongée) a été homogénéisé à la main avant le prélèvement des sous-échantillons. Dix réplicats de $2 \mathrm{~g}$ (poids humide) ont été prélevés par carotte sur trois carottes échantillonnées à RO et DA le 21 juin 1994, et sur le site PNOC le 24 février 1993. Ces dates ont été choisies car représentatives de concentrations extrêmes en Chl $a$ dans la colonne d'eau (maximum en rade et minimum en Manche). Le nombre minimal de sous-échantillons représentatifs de l'échantillon analysé a été déterminé selon Elliott [20]. Les moyennes et écarts types des concentrations en pigments ont été calculés entre deux, puis trois... puis les dix sous-échantillons. Le pourcentage d'erreur (D) sur la moyenne est estimé en fonction du nombre de réplicats par la formule $D=s /(x \cdot \sqrt{n})$, avec $s$ : écart type, $x$ : moyeme et $n$ : nombre de réplicals. $D$ diminue en fonction de l'augmentation progressive de la surface des sous-échantillons, pour atteindre un palier [20]. Le seuil de $20 \%$ préconisé en benthologie a été retenu pour déterminer le nombre minimal de réplicats représentatifs.

\subsection{Extraction des pigments}

Choix du solvant: l'acétone à $90 \%$ a été choisi comme solvant d'extraction car (1) c'est un solvant plus efficace que le méthanol $[6,40,64] ;$ (2) le déplacement du pic de phéopigments en milieu acide impliquerait une neutralisation supplémentaire ou une complication des calculs de concentration $[25,41,65] ;$; (3) les erreurs associées à des mélanges de pigments acidifiés à plus de $3 \times 10^{-3} \mathrm{M} \mathrm{HCL}$ sont plus faibles dans l'acétone que dans le méthanol [6, 57], la conversion de la Chl $a$ en phéo $a$ étant supérieure dans l'acétone à $90 \%$ [6] ; (4) les coefficients d'absorption spécifique des chlorophylles dans l'acétone à $90 \%$ sont connus $[2,61]$; (5) l'utilisation d'acétone à moins de 80 \% entraîne une dégradation de la Chl $a$ [54]; et (6) la présence de chlorophyllide $a$ dans les solutions extraites avec de l'acétone à $90 \%$ ne provoque pas d'interférences car elle a le même spectre et le même coefficient d'extinction que la Chl $a[45,59]$.

Protocole d'extraction : Il a été mis au point sur les échantillons de la rade (couche $0-5 \mathrm{~mm}$ ), pour lesquels deux procédures d'extraction, $\mathrm{P} 1$ et $\mathrm{P} 2$, ont été testées [3]. Dans P1, les réplicats ( $\mathrm{g}$ de poids humide) ont été homogénéisés avec $6 \mathrm{ml}$ d'acétone à $90 \%$, avant extraction à $4{ }^{\circ} \mathrm{C}$ pendant 16 à 20 heures [61]. Après centrifugation, le surnageant a été tilltré (membrane Téflon PTFE de porosité 0,45 ou $0,2 \mu \mathrm{m}$, volume final de $\pm 6 \mathrm{ml}$ ). Dans P2, trois cxtractions succcssives ont été réalisées : les réplicats ( $2 \mathrm{~g}$ de poids humide) ont été homogénéisés par trois fois avec $2 \mathrm{~mL}$ d'acétone à $90 \%$, centrifugés (2000 tr. $\mathrm{min}^{-1}$ pendant $5 \mathrm{~min}$ ) et le surnageant filtré. Les trois surnageants ont été mélangés et le volume total a été mesuré. Les extraits acétoniques ont été centrifugés à 3500 tr. $\mathrm{min}^{-1}$ pendant $5 \mathrm{~min}$ afin que la turbidité des solu- 
tions n'excède pas une densité optique (DO) de 0,01 à 0,05 de DO par centimètre de cuve $[2,25,42,61]$.

\subsection{Analyses pigmentaires}

Les analyses pigmentaires ont été réalisées 12 (rade de Brest) à 24 mois (Manche) après les prélèvements. Les concentrations en Chl $a$ et en phéo $a$ dans les extraits acétoniques ont été déterminées au spectrophotomètre SECOMAM S 1000 PC. Ce dernier a été étalonné en $D O$ à l'aide de trois filtres référence de 1,3 et $4 \mathrm{~mm}$ d'épaisseur, et en longueur d'onde à l'aide d'un filtre d'oxyde d'holmium (précision $\pm 0,5 \mathrm{~nm}$ [6]). La bande passante du spectrophotomètre n'excède pas $2 \mathrm{~nm}$ [2]. Les mesures ont été faites sur les extraits acétoniques $(6 \mathrm{~mL})$ avant et après acidification par $100 \mu \mathrm{L}$ HCL $0,1 \mathrm{~N}$ ([24, 59]; Riaux-Gobin, communication personnelle). Les concentrations en $\mathrm{Chl} a$ et phéo $a$ ont été calculées d'après les équations de Lorenzen [36] adaptées pour le sédiment $[52,55,60]$, en utilisant le rapport d'acidification recommandé pour les zones côtières $[6,15,39]$ ). L'ensemble des manipulations de sous-échantillonnage, d'extraction et d'analyse a été fait à l'obscurité pour prévenir les risques de photodégradation.

\section{RÉSULTATS}

\subsection{Nombre minimal de réplicats représentatifs de la variabilité intra-échantillons}

Le calcul de la raréfaction du pourcentage d'erreur sur la moyenne des concentrations $(D)$ réalisé sur dix réplicats provenant d'échantillons prélevés le 24 février 1993 (TV) et le 21 juin 1994 (RO, DA) montre une stabilisation de la variance à partir de cinq réplicats pour la Chl $a$ (RO : $6,3 \%$; DA : $14,7 \%$; TV : 18,9\%) et les phéo $a$ (RO : $10 \%$; DA : 9,6\%), au seuil de $20 \%$. Les mesures de phéo $a$ en Manche ne sont pas présentées en raison de l'obtention de valeurs négatives ; selon Brown et al. [6] et Lehman [31], elles résulteraient de la présence de produits de dégradation eVou de $\mathrm{Chl} c$. Le nombre minimal de réplicats représentatif de l'hétérogénéité intra-échantillon a donc été fixé à cinq. Pour les échantillons de la rade, et en accord avec la littérature $[6,7,12,27,29,30$, $47,53,67]$, cinq réplicats ont été prélevés par tranche de $5 \mathrm{~mm}$ dans les trois premiers centimètres du sédiment (soit 30 réplicats par carotte au total). En Manche, cinq réplicats au total ont été effectués par échantillon, faute de pouvoir sous-échantillonner sur la verticale.

\subsection{Comparaison des techniques d'extraction}

La procédure usuelle d'extraction des pigments chlorophylliens dans l'eau est une extraction à froid avec de l'acétone à $90 \%$, en une seule étape, i.e. en laissant les échantillons au réfrigérateur pendant 16 à 20 heures [24, 25, 61, 69]. Dans le cas d'échantillons de sédiment cependant, l'extraction à froid (P1) serait moins efficace qu'une procédure d'extraction instantanée (P2) en trois étapes et à température ambiante [3]. Une étude comparative des deux procédures a été menée sur des carottes prélevées à SA le 12 avril 1995, pour la mise au point du protocole. Seule la couche superficielle $(0-5 \mathrm{~mm}) \mathrm{du}$ sédiment a été analysée, dans la mesure où elle contient généralement les plus fortes teneurs en pigments [3, 29, $47,53,67]$, et en moyenne $75 \%$ des pigments totaux [7]. Il s'est avéré par la suite que cette tendance n'était pas respectée ici, SA étant seul à présenter une couche sédimentaire de surface plus riche en pigments chlorophylliens ( $21 \%$ des pigments totaux en moyenne). Sur dix réplicats, cinq ont été traités par extraction à froid $(\mathrm{P} 1)$ et les cinq autres par extraction instantanée (P2). I.es valeurs moyennes des concentrations en $\mathrm{Chl}$ a sont: $1,63 \pm 0,44 \mu \mathrm{g} \mathrm{g}^{-1}$ PS (poids sec) avec P1, et 2,31 \pm $0,38 \mu \mathrm{g} \mathrm{g}^{-1}$ PS avec P2. Une extraction plus complète de Chl $a$ est donc obtenue avec P2 (P2/P1 =1,42). A titre comparatif, un rapport $\mathrm{P} 2 / \mathrm{P} 1$ moyen de 1,2 a été calculé par Barlow et al. [3] sur des échantillons prélevés entre 7 et $10 \mathrm{~m}$ dc profondcur dans un marais salant. La procédure d'extraction instantanée a donc été retenue pour cette étude.

\section{3. Évolution temporelle des teneurs en pigments du sédiment}

$S A$ : en 1994, la couche superficielle du sédiment $(0-$ $5 \mathrm{~mm}$ ) présente deux pics de concentration en Chl $a$, les 21 juin et 18 août ( 3 et $2 \mu \mathrm{g} \mathrm{g}^{-1} \mathrm{PS}$, respectivement ; figure 2). Un gradient de concentration décroissant sur la verticale est observé pour la $\mathrm{Chl} a$ (facteur 3 entre les couches $0-5 \mathrm{~mm}$ et $25-30 \mathrm{~mm}$ le 21 juin). Le pic de chlorophylle du 21 juin dans les sédiments superficiels n'est pas observé dans les tranches $20-25$ et $25-30 \mathrm{~mm}$. De même, le pic de Chl $a$ dans la couche $0-5 \mathrm{~mm}$ le 18 août ne se retrouve pas dans les couches sédimentaires plus profondes, alors qu'une augmentation des teneurs en 


\section{Sainte-Anne - 1994}

Chla

$0-5 \mathrm{~mm}$

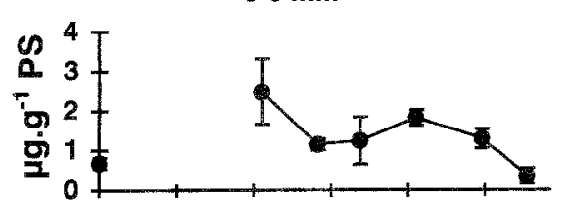

5-10 mm

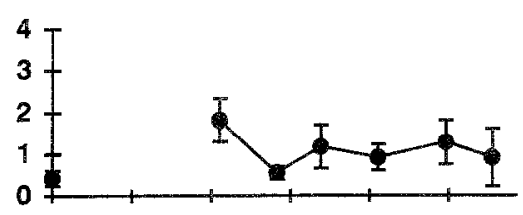

$10-15 \mathrm{~mm}$

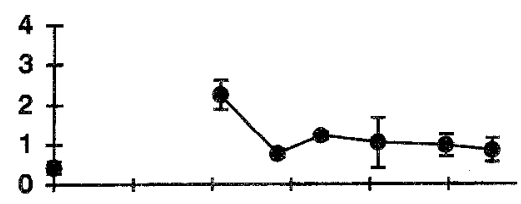

$15-20 \mathrm{~mm}$

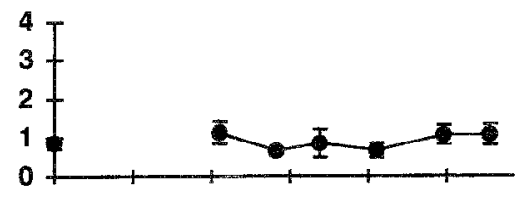

20-25 mm

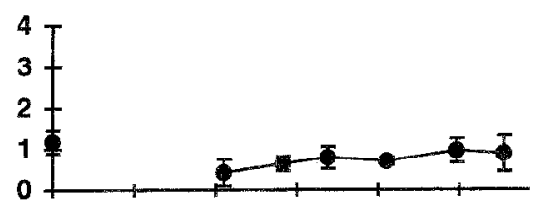

25-30 mm

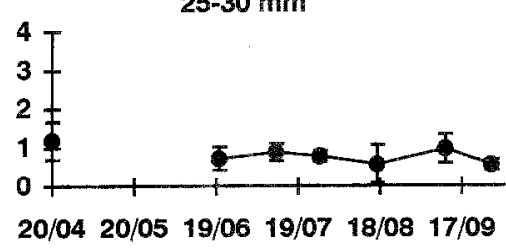

Phéo a

$0-5 \mathrm{~mm}$

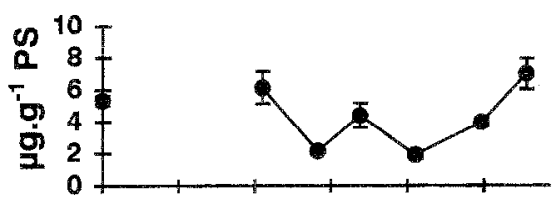

$5-10 \mathrm{~mm}$

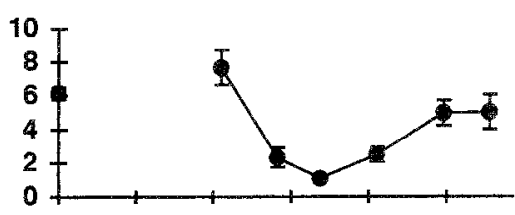

10-15 mm

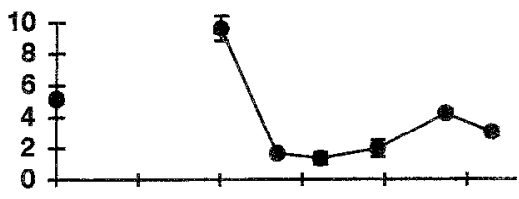

$15-20 \mathrm{~mm}$

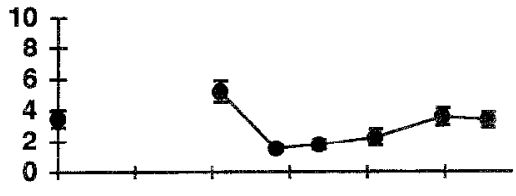

20-25 mm

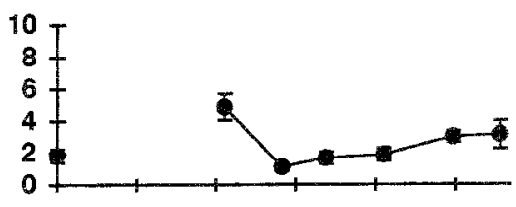

25-30 mm

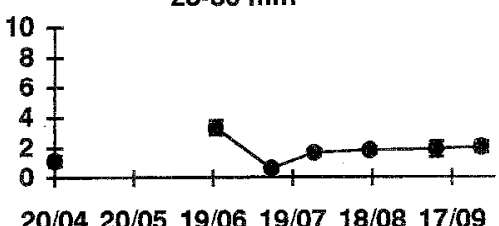

Figure 2. Sainte-Anne (SA). Concentrations en chlorophylle $a$ et en phéophytine a ( $\mu \mathrm{g} \cdot \mathrm{g}^{-1} \mathrm{PS}$, sédiment sec ; moyennes \pm SD) dans les trois premiers centimètres du sédiment en 1994, mesurées par spectrophotométrie après une extraction de type P2.

Figure 2. Sainte-Anne (SA). Chlorophyll $a$ and phaeophytin $a$ concentrations ( $\mu \mathrm{g} \cdot \mathrm{g}^{-1} \mathrm{DW}$, sediment dry weight; mean $\pm \mathrm{SD}$ ) in the top $3 \mathrm{~cm}$ of sediment in 1994 (spectrophotometry after extraction procedure P2). 
phéo $a$ est notée en septembre entre 5 et $25 \mathrm{~mm}$. Ce résultat indiquerait que le microphytobenthos vivant est surtout localisé dans les sédiments les plus superficiels, alors que la dégradation pigmentaire sur la verticale résulterait du piégeage de cellules algales mortes dans les couches sédimentaires plus profondes. Trois pics de phéo $a$ sont observés dans la tranche $0-5 \mathrm{~mm}$ les 21 juin $\left(6 \mu \mathrm{g} \mathrm{g}^{-1}\right.$ PS), 28 juillet ( $4 \mu \mathrm{g} \mathrm{g}^{-1}$ PS) et 29 septembre $\left(6 \mu \mathrm{g} \mathrm{g}^{-1}\right.$ PS). Le pic du 21 juin est observé dans l'ensemble des couches sédimentaires $\left(5\right.$ à $10 \mu \mathrm{g} \mathrm{g}^{-1}$ PS entre 0 et $25 \mathrm{~mm}$ ), contrairement aux deux autres pics. Les pics de Chl $a$ et de phéo $a$ dans le sédiment sont associés à des pics de concentrations pigmentaires à l'interface eausédiment (eau surnageante des carottes) les 21 juin ( $8 \mu \mathrm{g}$ $\mathrm{Chl} a \mathrm{~L}^{-1}$ et $34 \mu \mathrm{g}$ phéo $\left.a \mathrm{~L}^{-1}\right)$ et 29 septembre $(37,3 \mu \mathrm{g}$ phéo $a \mathrm{~L}^{-1}$ ).

$D A$ : Pour la période d'étude considérée (avril à août), l'évolution des concentrations en pigments (figure 3) montre des variations temporelles semblables à celles observées à SA pour la chlorophylle, excepté dans les couches profondes du sédiment ( 20 à $30 \mathrm{~mm}$ ). A l'exception du 12 juillet, la concentration moyenne en $\mathrm{Chl} a$ est de l'ordre de 2 à $3 \mu \mathrm{g} \mathrm{g}^{-1}$ PS entre la mi-mai et la mi-août, avec une évolution temporelle identique dans toutes les couches sédimentaires et un gradient de concentration sur la verticale peu marqué. Les concentrations en phéo $a$ présentent, comme à $\mathrm{SA}$, un maximum le 21 juin dans toutes les tranches sédimentaires. Les teneurs en phéo $a$ à cette période (20-32 $\mu \mathrm{g} \mathrm{g}^{-1}$ PS sur l'ensemble des couches sédimentaires ; figure 3) sont trois à cinq fois plus élevées que celles mesurées à SA à la même date. L'absence de gradient de concentration en phéopigments, décroissant sur la verticale, se traduit par une évolution temporelle des profils sédimentaires similaire entre 0 et $30 \mathrm{~mm}$, contrairement à SA. Les concentrations pigmentaires à l'interface eau-sédiment sont maximales le 19 mai (106 $\mu \mathrm{g} \mathrm{Chl} a \mathrm{~L}^{-1}$ et $543 \mu \mathrm{g}$ phéo $a \mathrm{~L}^{-1}$ ), elles présentent un pic secondaire le 21 juin $\left(77 \mu \mathrm{g}\right.$ phéo $\left.a \mathrm{~L}^{-1}\right)$, et elles sont minimales en juillet-août $\left(<0,1 \mu \mathrm{g} \mathrm{L}{ }^{-1}\right.$ de $\mathrm{Chl} a$ et phéo $a$ ).

$R O$ : les concentrations moyennes en Chl $a$ sur ce site $\left(<1,5 \mu \mathrm{g} \mathrm{g}^{-1} \mathrm{PS}\right.$; figure 4) sont inférieures à celles obtenues à SA et à DA. L'évolution temporelle des teneurs en Chl $a$ est variable suivant les couches sédimentaires : dans les sédiments superficiels $(0-10 \mathrm{~mm})$, une floraison printanière est observée le 19 mai $\left(0,8-1,3 \mu \mathrm{g} \mathrm{g}^{-1}\right.$ PS, avec la présence d'un gradient de concentration décroissant sur la verticale entre 5 et $30 \mathrm{~mm}$ à cette date), alors que les couches entre 10 et $30 \mathrm{~mm}$ présen- tent un maximum de concentration le 20 juin. L'évolution estivale des teneurs en Chl $a$ est plus complexe, avec un pic secondaire de concentration le 19 août entre 20 et $30 \mathrm{~mm}\left(0,73\right.$ à $0,89 \mu \mathrm{g} \mathrm{g}^{-1}$ PS $)$, contrairement aux couches de surface qui présentent une augmentation progressive des teneurs en août et septembre. La phéo a montre trois pics de concentration, dans la couche superficielle du sédiment, les 19 mai $\left(15 \mu \mathrm{g} \mathrm{g}^{-1}\right.$ PS), 8 juillet ( $10 \mu \mathrm{g} \mathrm{g}^{-1}$ PS) et 13 septembre $\left(13 \mu \mathrm{g} \mathrm{g}^{-1}\right.$ PS ; figure 4). Le pic printanier est observé dans toutes les couches sédimentaires, avec un gradient de concentration décroissant sur la verticale $(-41 \%)$. RO se démarque ainsi des deux sites peu profonds avec une biomasse pigmentaire surtout localisée dans les sédiments les plus superficiels. Les pics printaniers de concentration pigmentaire sont synchrones dans le sédiment et à l'interface eau-sédiment : les pics de phéo a (19 mai) et de Chl $a$ (20/06) dans le sédiment sont associés à des pics similaires dans l'eau surnageante des carottes $\left(87 \mu \mathrm{g}\right.$ phéo $a \mathrm{~L}^{-1}$ le 19 mai et $4,8 \mu \mathrm{g} \mathrm{Chl} a \mathrm{~L}^{-1}$ le 20 juin). En revanche, aucune correspondance n'est observée entre les fortes concentrations pigmentaires dans l'eau surnageante en juillet (12 et $13,6 \mu \mathrm{g} C h 1 a \mathrm{~L}^{-1}$ et 95 et $67 \mu \mathrm{g}$ phéo $a \mathrm{~L}^{-1}$ les 8 et 28 juillet, respectivement) et les teneurs pigmentaires du sédiment (figure 4).

TV (Manche) : l'impossibilité de découper les carottes du Reineck en sections sur la verticale ne permet qu'une étude globale du contenu pigmentaire des échantillons, après homogénéisation manuelle de l'ensemble du sédiment de chaque prélèvement. De plus, l'utilisation du carottier Reineck n'a pas permis l'étude de l'interface eau-sédiment (eau surnageante), en raison des perturbations occasionnées lors des carollages (élimination de la pellicule superficielle du sédiment par vannage) ou à la remontée (remise en suspension du sédiment liée à la surface de contact trop grande). Les variations temporelles de concentration en pigments dans le sédiment (figure 5) ont une amplitude moindre, comparativement aux sites côtiers de la rade de Brest. Un facteur 3 est observé en 1993 dans l'évolution saisonnière des teneurs en Chl $a$, contre un facteur 3,5 pour les variations en phéo $a$. Les écarts types associés aux valeurs moyennes de concentration traduisent une hétérogénéité intra- et/ou interéchantillons relativement grande. Les teneurs en Chl $a$ présentent un pic de concentration le 15 mars $\left(1,58 \mu \mathrm{g} \mathrm{g}^{-1}\right.$ PS), alors que les teneurs en phéo $a$ ne montrent pas de pic de concentration entre le 13 avril et le 8 novembre $\left(0,65\right.$ à $0,82 \mu \mathrm{g} \mathrm{g}^{-1}$ PS). 


\section{Ducs d'Albe $=1994$}

Chla

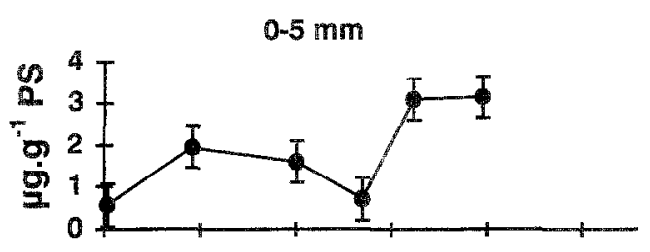

5-10 mm

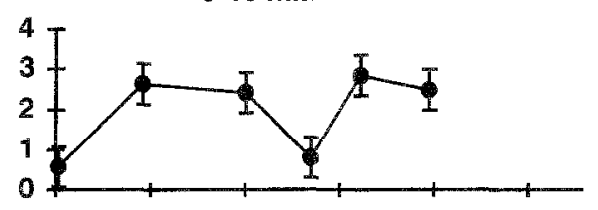

10-15 mm

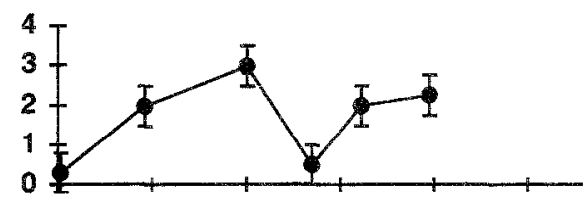

$15-20 \mathrm{~mm}$

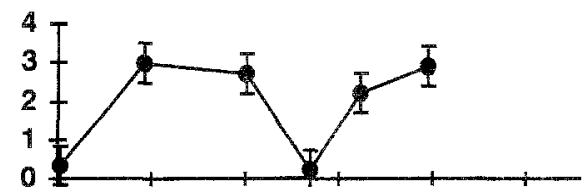

$20-25 \mathrm{~mm}$
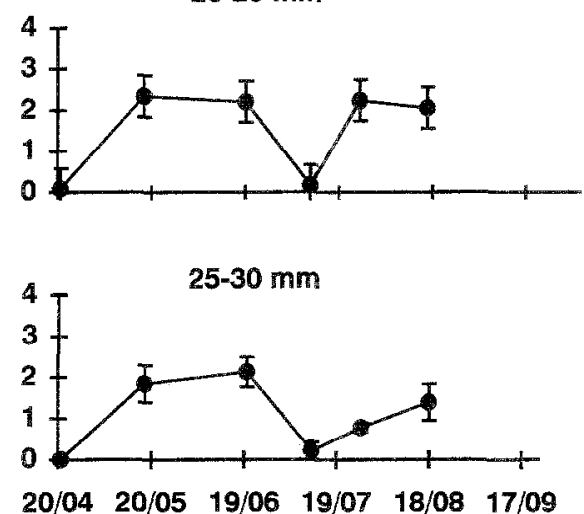

Phéo a

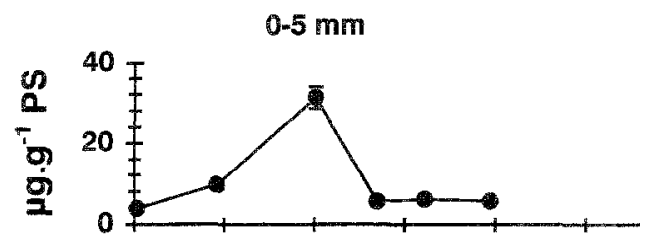

5-10 mm

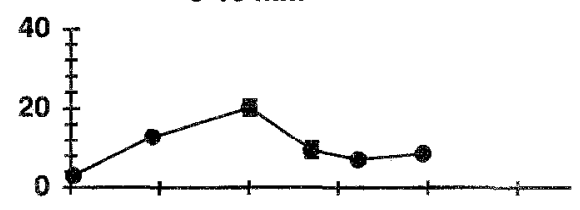

$10-15 \mathrm{~mm}$

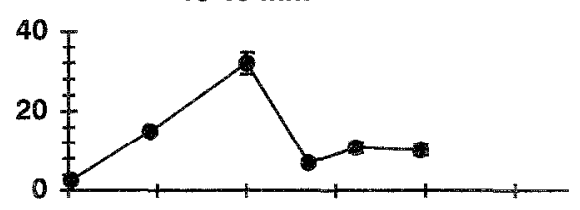

$15-20 \mathrm{~mm}$

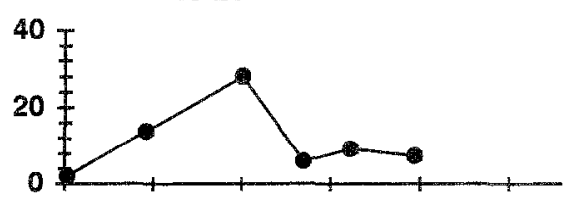

20-25 mm
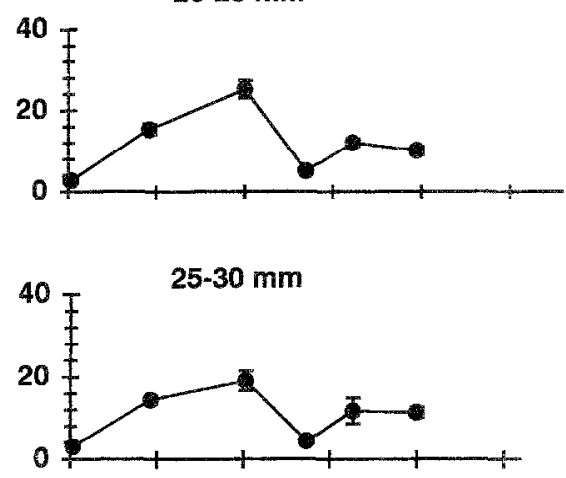

$20 / 0420 / 05 \quad 19 / 06 \quad 19 / 07 \quad 18 / 08 \quad 17 / 09$

Figure 3. Ducs d'Aibe (DA). Concentrations en chlorophylle $a$ et en phéophytine a ( $\mu$ g.g ${ }^{-1}$ PS, sédiment sec; moyennes \pm SD) dans les trois premiers centimètres du sédiment en 1994, mesurées par spectrophotométrie après une extraction de type P2.

Figure 3. Ducs d'Albe (DA). Chlorophyll $a$ and phaeophytin $a$ concentrations ( $\mu \mathrm{g} \cdot \mathrm{g}^{-1} \mathrm{DW}$, sediment dry weight, mean $\pm \mathrm{SD}$ ) in the top $3 \mathrm{~cm}$ of sediment in 1994 (spectrophotometry after extraction procedure P2). 


\section{Roscanvel - 1994}

Chla

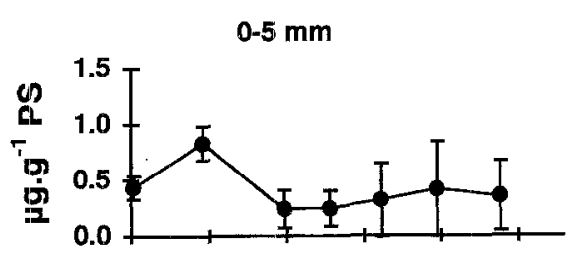

5-10 mm

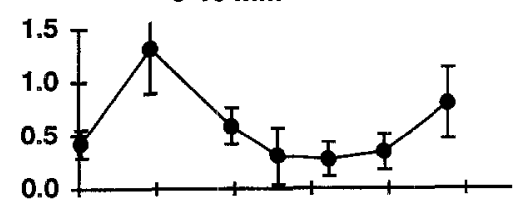

10-15 mm

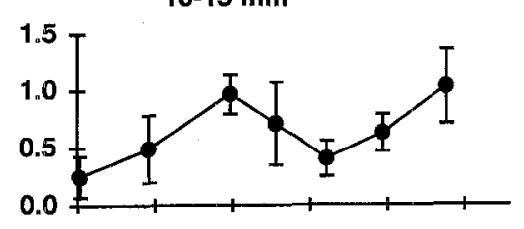

$15-20 \mathrm{~mm}$

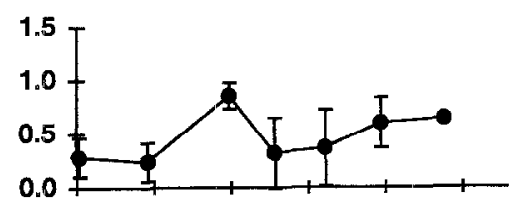

20-25 mm
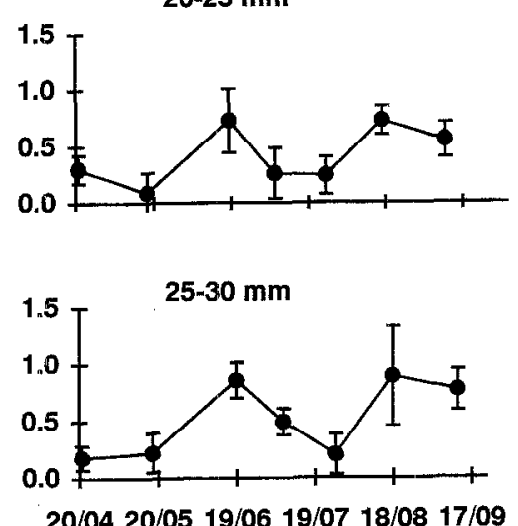

Phéo a

$0-5 \mathrm{~mm}$

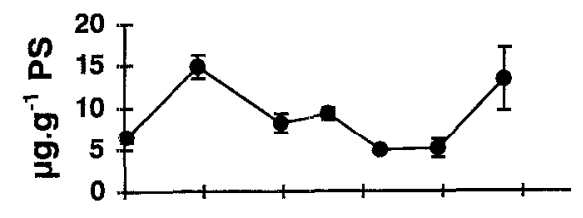

$5-10 \mathrm{~mm}$

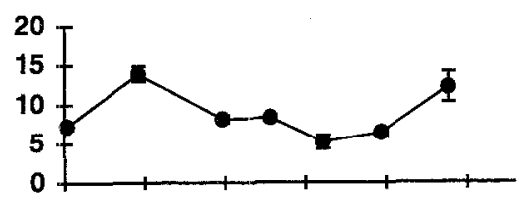

$10-15 \mathrm{~mm}$

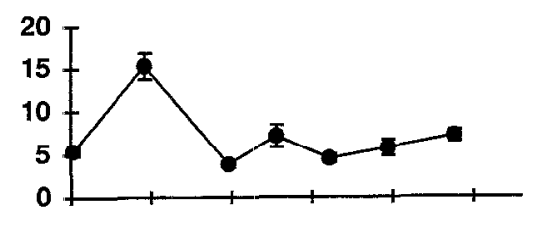

$15-20 \mathrm{~mm}$

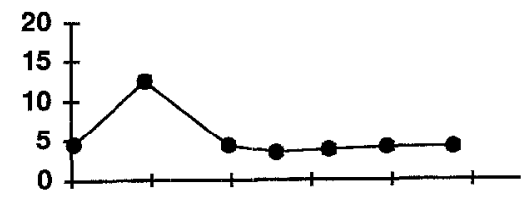

20-25 mm

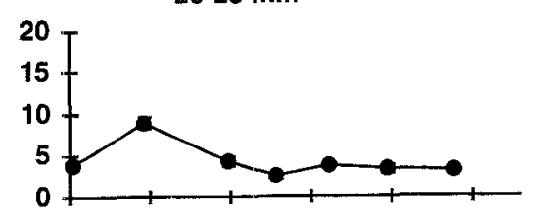

25-30 mm

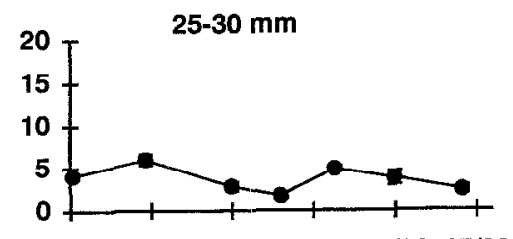

20/04 20/05 19/06 19/07 18/08 17/09

Figure 4. Roscanvel (RO). Concentrations en chlorophylle a et en phéophytine $a\left(\mu \mathrm{g} . \mathrm{g}^{-1} \mathrm{PS}\right.$, sédiment sec ; moyennes $\left.\pm \mathrm{SD}\right)$ dans les trois premiers centimètres du sédiment en 1994, mesurées par spectrophotométrie après une extraction de type P2.

Figure 4. Roscanvel (RO). Chlorophyll $a$ and phaeophytin $a$ concentrations ( $\mu \mathrm{g}_{\mathrm{g}} \mathrm{g}^{-1} \mathrm{DW}$, sediment dry weight; mean $\left.\pm \mathrm{SD}\right)$ in the top $3 \mathrm{~cm}$ of sediment in 1994 (spectrophotometry after extraction procedure P2). 


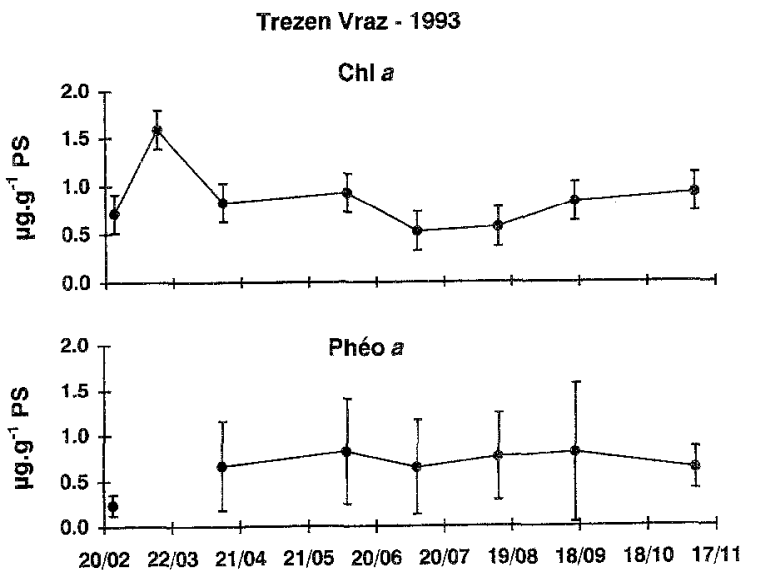

Figure 5. Trezen Vraz (TV). Concentrations en chlorophylle $a$ et en phéophytine a ( $\mu \mathrm{g} \cdot \mathrm{g}^{-1} \mathrm{PS}$, sédiment sec) du sédiment en 1993, mesurées par spectrophotométrie après une extraction de type P2 (moyennes $\pm \mathrm{SD}$ de quatre échantillons par date et de cinq réplicats par échantillon)

Figure 5. Trezen Vraz (TV). Chlorophyll $a$ and phaeophytin $a$ concentrations ( $\mu \mathrm{g} \cdot \mathrm{g}^{-1} \mathrm{DW}$; sediment dry weight) in sediment samples in 1993 (spectrophotometry after extraction procedure P2; means \pm SD of 4 samples per date and 5 replicates per sample).

\subsection{Estimation de la biomasse chlorophyllienne}

Les calculs de biomasse chlorophyllienne moyenne (en $\mathrm{g} \mathrm{C} \mathrm{m}^{-2}$; figure 6) ont été effectués à partir des concentrations en $\mathrm{Chl} a$, sommées sur les différentes sections dans le cas de la rade de Brest, et converties en poids de carbone organique par le facteur de conversion $\mathrm{C} / \mathrm{Chl}$ $a=40$ [16]. La biomasse chlorophyllienne montre deux pics à SA les 21 avril (floraison printanière; $84,3 \mathrm{mg} \mathrm{Chl}$ $a \mathrm{~m}^{-2}$ soit $3,4 \mathrm{~g} \mathrm{C} \mathrm{m}^{-2}$ ) et 12 septembre (floraison automnale ; 92,4 $\mathrm{mg} \mathrm{Chl} a \mathrm{~m}^{-2}$ soit 3,7 $\mathrm{g} \mathrm{C} \mathrm{m}^{-2}$ ), et des valeurs relativement stables en juillet-août (de 35,5 à $46,3 \mathrm{mg}$ Chl $a \mathrm{~m}^{-2}$ soit 1,4 à $1,9 \mathrm{~g} \mathrm{C} \mathrm{m}^{-2}$ ). Elle présente également un pic printanier à DA (19 mai ; $112,8 \mathrm{mg} \mathrm{Chl} a \mathrm{~m}^{-2}$ soit $\left.4,5 \mathrm{~g} \mathrm{C} \mathrm{m}^{-2}\right)$ et un maximum secondaire estival $(77,5 \mathrm{mg}$ Chl $a \mathrm{~m}^{-2}$ soit $3,1 \mathrm{~g} \mathrm{C} \mathrm{m}^{-2}$ ). DA présente les plus fortes variations temporelles de biomasse chlorophyllienne parmi les quatre sites étudiés. A RO, un pic principal est observé le 13 septembre $\left(64,8 \mathrm{mg}\right.$ Chl $a \mathrm{~m}^{-2}$ soit $2,6 \mathrm{~g} \mathrm{C} \mathrm{m}^{-2}$ ) ; la biomasse chlorophyllienne présente peu de variations entre le 19 mai et le 8 juillet (de 28,7 à $34,1 \mathrm{mg} \mathrm{Chl} a \mathrm{~m}^{-2}$ soit 1,1 à $1,4 \mathrm{~g} \mathrm{C} \mathrm{m}^{-2}$ ). En particulier, le pic de biomasse observé le 19 mai à $\mathrm{DA}$ ne se retrouve pas à RO. Globalement, les pics de biomasse chlorophyllienne présenteraient un décalage entre ces deux sites (e.y., le minimuin estival est observé deux semaines plus tard à RO, comparativement à DA). Trois pics saisonniers sont notés à TV les 15 mars $\left(214,6 \mathrm{mg} \mathrm{Chl} a \mathrm{~m}^{-2}\right.$ soit

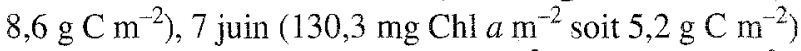
et 16 septembre $\left(114,5 \mathrm{mg} \mathrm{Chl} a \mathrm{~m}^{-2}\right.$ soit $4,6 \mathrm{~g} \mathrm{C} \mathrm{m}^{-2}$; figure 6).

La comparaison rade/Manche est hasardeuse en raison de l'utilisation de protocoles d'échantillonnage et d'analyse différents, d'années de suivi différentes (variabilité interannuelle) et d'une dégradation éventuelle du stock pigmentaire lors de la congélation potentiellement variable (liée à la durée de stockage des échantillons). Les faibles valeurs de biomasse chlorophyllienne calculées en rade de Brest pourraient illustrer l'intensité du broutage des microphytes par la faune benthique (rapport $\mathrm{Chl}$ a/phéo $a$ $<0,5$; figures 2 à 4 ). Par comparaison, les échantillons du TV se caractérisent par un stock pigmentaire moins dégradé (rapport $\mathrm{Chl}$ a/phéo $a \geq 1$; figure 5 ). Globalement, la biomasse pigmentaire totale ( $\mathrm{Chl} a+$ phéo $a$ ) dans les sédiments est deux à quatre fois supérieure en rade de Brest, comparativement à la Manche occidentale (SA : 131,3 à 477,6 $\mathrm{mg} \mathrm{m}^{-2}$; DA : 246,8 à 934,4 $\mathrm{mg} \mathrm{m}^{-2}$; RO : 159,5 à $668,7 \mathrm{mg} \mathrm{m}^{-2}$; TV : 88,2 à $254,4 \mathrm{mg} \mathrm{m}^{-2}$ ). Les moyennes annuelles de la biomasse pigmentaire totale montrent un facteur 3,4 entre TV $(152,4 \pm$ $\left.28,1 \mathrm{mg} \mathrm{m}^{-2}\right)$ et DA $\left(512,0 \pm 274,4 \mathrm{mg} \mathrm{m}^{-2}\right)$, contre un facteur 3,1 avec RO $\left(468,5 \pm 222,5 \mathrm{mg} \mathrm{m}^{-2}\right)$ et un facteur 1,9 avec SA $\left(296,7 \pm 138,9 \mathrm{mg} \mathrm{m}^{-2}\right)$. Ces variations illustrent le gradient de biomasse décroissant de l'estuaire vers la mer ouverte et l'impact de la profondeur des sites.

\section{DISCUSSION}

\subsection{Biomasse chlorophyllienne}

Le calcul de biomasses chlorophylliennes en poids de carbone organique $(\mathrm{CO})$ à partir d'un facteur de conversion $\mathrm{C} / \mathrm{Chl} a$ constant est très aléatoire dans la mesure où ce rapport varie avec les populations de microphytes considérées et, pour une population donnée, l'état physiologique des cellules et les facteurs climatiques locaux. Une étude comparative de différentes populations de diatomées benthiques dans l'estuaire du Ems-Dollard entre 1976 et 1978 montre une variation intra- et interannuelle du rapport $\mathrm{C} / \mathrm{Chl} a$ de 10,2 à 153,9 avec des moyennes annuelles de 40,3 en 1976, 41,2 en 1977, et 61,4 en 1978 [16]. En rade de Brest, l'existence de populations microphytobenthiques à Chlorophyceae dans l'anse du Fret 

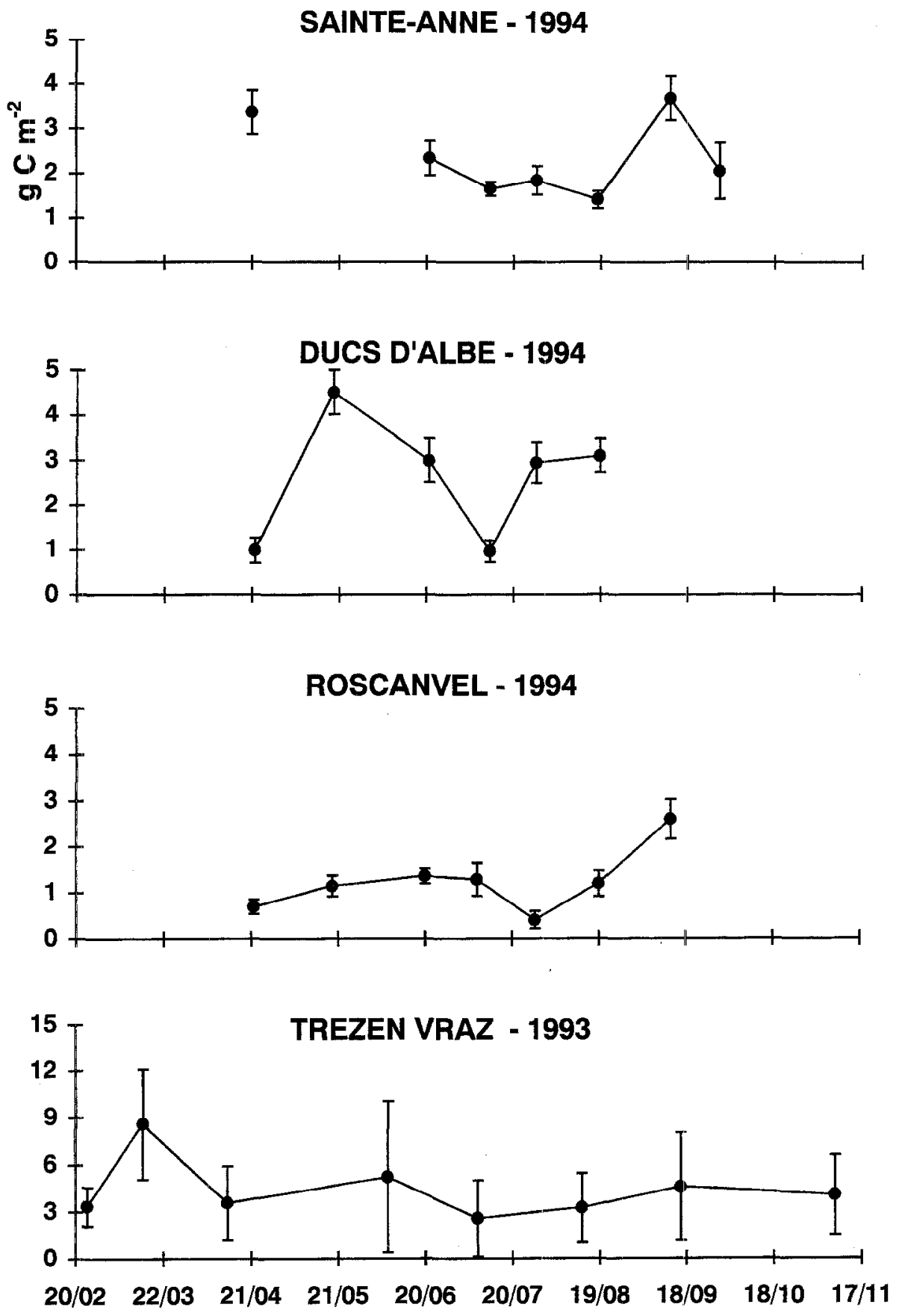

Figure 6. Biomasse chlorophyllienne des sédiments en carbone organique $\left(\mathrm{g} \mathrm{C} \mathrm{m}^{-2}\right)$ calculée à partir des concentrations en chlorophylle $a$ et du rapport C/Chl $a=40$ (d'après De Jonge, 1980).

Figure 6. Chlorophyll biomass in organic carbon $\left(\mathrm{g} \mathrm{C} \mathrm{m}^{-2}\right)$ calculated from sediment chlorophyll $a$ concentrations and the ratio $\mathrm{C} / \mathrm{Chl} a=40$ (from De Jonge, 1980). 
[53] ne permet pas de préjuger de la nature des populations rencontrées sur nos sites ateliers. Dans cette étude, les biomasses en Chl $a$ ont été converties en poids de $\mathrm{CO}$ à partir d'un rapport C/Chl $a=40[12,16]$, facteur également utilisé pour les populations phytoplanctoniques de la rade de Brest [26]. La conversion en CO a été établie dans le but de modéliser ultérieurement le cycle du carbone à méso-échelle ; par contre, les biomasses chlorophylliennes obtenues dans cette étude sont comparées à celles de la littérature en termes de teneurs en Chl $a$ du sédiment, de manière à faire abstraction des biais inhérents à l'emploi de facteurs de conversion.

Les biomasses chlorophylliennes des sédiments sableux en rade de Brest (10-113 $\mathrm{mg} \mathrm{Chl} \mathrm{a} \mathrm{m^{-2 }}$; moyennes annuelles entre 39 et $65 \mathrm{mg} \mathrm{Chl} a \mathrm{~m}^{-2}$ ) sont deux à trois fois supérieures à celles trouvées, pour les sédiments sableux subtidaux (prof $\leq 30 \mathrm{~m}$ ), en baie de Douarnenez (15-55 mg Chl $a \mathrm{~m}^{-2}$ [5]), en baie de Morlaix (25-60 mg Chl $a \mathrm{~m}^{-2}$; Riaux-Gobin et al., non publié) et en Méditerranée (24-64 $\mathrm{mg} \mathrm{Chl} a \mathrm{~m}^{-2}$ [13]). Elles sont du même ordre de grandeur que dans le Mississippi Sound sous une végétation à Halodule wrightii $\left(\leq 125 \mathrm{mg} \mathrm{Chl} a \mathrm{~m}^{-2}\right.$ [14]). Une étude antérieure sur les vases sableuses de la rade de Brest a fourni des concentrations en $\mathrm{Chl} a$ dans la couche $0-5 \mathrm{~mm}$ très supérieures à celles obtenues dans cette étude (22 à $26 \mu \mathrm{g} \mathrm{g}^{-1}$ PS lors des floraisons printanières, dosages HPLC et fluorimétriques [53]).

Cependant, les teneurs maximales en phéo $a$ de la couche 0-5 mm du sédiment dans l'anse du Fret $\left(19 \mu \mathrm{g} \mathrm{g}^{-1}\right.$ PS ; dosage fluorimétrique) sont du même ordre de grandeur que celles obtenues à RO, et inférieures de $50 \%$ à celles mesurécs à DA. L'anse du Fret, situće dans le bassin sud de la rade (figure I), comprend des sédiments vaseux subtidaux peu profonds $(15 \mathrm{~m})$ exposés à une circulation résiduelle de marée tourbillonnaire. Bien que les conditions environnementales soient globalement différentes de celles régnant sur nos sites d'études (profondeur moindre, envasement supérieur du sédiment), cette zone et RO présentent des caractéristiques hydrodynamiques communes. On ne peut éliminer l'hypothèse d'une sous-estimation des biomasses chlorophylliennes par dégradation pigmentaire liée à une conservation trop longue (12 à 18 mois à $-20^{\circ} \mathrm{C}$ ) des échantillons avant analyse.

Cependant, les faibles valeurs du rapport Chl $a$ /phéo $a$ en rade (à DA notamment) pourraient également résulter du broutage des microphytes par la faune benthique ou du piégeage de détritus macrophytes dans les sédiments $[7$, 11]. Si les valeurs de biomasse chlorophyllienne sur le Trezen Vraz (64 à $215 \mathrm{mg} \mathrm{Chl} a \mathrm{~m}^{-2}$ ) sont supérieures à celles de la rade de Brest, la biomasse pigmentaire totale $($ Chl $a+$ phéo $a$ ) est deux à quatre fois plus élevée dans les sédiments de la rade. Ce résultat traduit la différence entre une baie côtière peu profonde eutrophe $[22,33,48$, 49] et une mer ouverte moins soumise aux apports anthropiques [50]. Les biomasses pigmentaires mesurées en rade de Brest sont cependant inférieures à celles observées dans les systèmes eutrophisés [62], confortant ainsi les conclusions de Ragueneau et al. [49] et Le Pape et al. [33] selun lesquelles la rade constitue un milieu eutrophe mais non eutrophisé.

La comparaison intersites de la biomasse chlorophyllienne dans la couche sédimentaire de surface permet d'axer l'étude sur la zone la plus productive du sédiment $[3,7,29,47,52-54,67]$, et directement sous l'influence du couplage pélagos-benthos. Les biomasses chlorophylliennes calculées en rade de Brest dans la couche $0-5 \mathrm{~mm}$

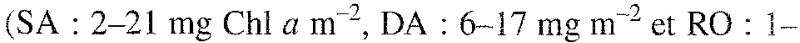
$10 \mathrm{mg} \mathrm{m}^{-2}$ ) restent très inférieures à celles mesurées par spectrophotométrie dans les vases et les sables subtidaux (prof. 8-15 m) de la baie de Laholm (couche 0-5 mm , 78 à $87 \mathrm{mg} \mathrm{m}^{-2}$ [62]. Ces résultats reflètent pro parte la variabilité spatiale liée à la nature sédimentaire des fonds. Les vases présentent généralement des biomasses pigmentaires supérieures à celles mesurées dans les sables (e.g. couche $0-5 \mathrm{~mm}$ des slikkes du Nord Finistère : 25 à $250 \mathrm{mg}$ Chl $a \mathrm{~m}^{-2}[52,54]$. La stabilité d'une vase permet une colonisation rapide par les populations de microphytes lors des périodes de fort éclairement, alors que l'instabilité supérieure d'un sable (en raison du mélange turbulent) limite la croissance des microalgues à la surface du sédiment [53].

Les variations observées intègrent également la variabilité résultant de l'emploi de protocoles d'échantillonnage et d'analyse pigmentaire différents. Les études comparatives sur la fiabilité relative des techniques d'analyse pigmentaire dans le sédiment sont contradictoires. Daemen [15] indique des variations dans les mesures de Chl a par spectrophotométrie (SP) et HPLC comprises entre 3 et $20 \%$ en moyenne et n'excédant jamais $30 \%$ en zone estuaricnne.

En revanche, Brown et al. [6] ne trouvent pas de différence significative entre les deux techniques. Sclon ces auteurs et Delgado et al. [19], la spectrophotométrie serait plus fiable que la fluorimétrie (F) dans le cas d'échantillons de sédiment, mais Daemen [15] trouve un rapport SP/F voisin de $1(1,06)$ en zone estuarienne. Les variations observées entre les techniques de dosage peuvent avoir pour origine une conversion incomplète de 
Chl $a$ en phéo $a$, ou la phéophytinisation de chlorophylles autres que Chl a [25]. En particulier, les spectres d'absorption dans la région rouge de la $\mathrm{Chl} b$ et de la phéophytine $b$ étant voisins de ceux de la Chl $a$ et de la phéophytine $a$, la Chl $a$ serait surestimée dans les dosages fluorimétriques ou spectrophotométriques lorsque le rapport $\mathrm{Chl} b / \mathrm{Chl} a$ dépasse $0,4[1,2,15,25,34,38,39$, $42,54,57,66]$. La présence de Chl $c$ dans les échantillons entraînerait une surestimation de $10 \%$ de la concentration en Chl $a$ lorsque le rapport $\mathrm{Chl} c / \mathrm{Chl} a$ est $>1[1$, $31,34,43]$, et une sous-estimation des teneurs en phéo $a$ [37].

D'autre part, les produits de dégradation à radicaux $M g$ libres, les caroténoïdes, les fucoxanthines et les $\beta$-carotènes peuvent aussi interférer dans la mesure de la $\mathrm{Chl} a$ $[24,31,57]$. Dans le cas de sédiments côtiers, la surestimation des teneurs en Chl $a$ proviendrait pro parte d'interférences liées à la présence de composés non phorbides $[6,15]$. Des substances humiques, des acides organiques [21], et le contenu en MO des échantillons de seston [17] sont également cités comme sources de variations. La spectrophotométrie ne permet pas de quantifier précisément les teneurs en chlorophylles $b$ et $c$, ni celles des produits de dégradation. On peut supposer que l'ensemble de ces pigments accessoires a contribué à introduire un biais dans nos mesures. L'interférence liée à la présence éventuelle de ces pigments dans les sédiments de la rade de Brest n'expliquerait cependant pas les fortes teneurs en phéopigments mesurées dans cette étude.

\subsection{Variabilité spatio-temporelle des teneurs en pigments}

L'absence de mesures de concentrations en sels nutritifs dans l'eau interstitielle ne permet pas de statuer sur une limitation éventuelle de la biomasse microphytobenthique par le stock de sels nutritifs. Les augmentations printanières de la température et de l'éclairement seraient à l'origine des floraisons microphytobenthiques dans la rade de Brest (Le Corre, communication personnelle). En termes de porosité du sédiment, les quatre sites d'études présentent des sédiments hétérogènes, à fraction grossière dominante (35 à $70 \%$ PS), propices à la circulation de l'eau interstitielle, à la bioturbation du sédiment par la faune benthique et aux migrations verticales actives du microphytobenthos [4]. Ces caractéristiques permettraient le développement des microphytes dans les trois sites de la rade $[7,58]$. En revanche, l'éclairement serait limitant à RO (biomasses chlorophylliennes les plus fai- bles), site le plus profond et soumis à une circulation résiduelle anticyclonique favorisant le dépôt de particules fines à la surface du sédiment. Cette hypothèse cst en accord avec les résultats de Ragueneau et al. [50] sur les facteurs contrôlant la production primaire pélagique dans cette zone.

L'étude du rôle de l'alternance vive-eau/morte-eau sur la dynamique du phytoplancton a mis en évidence le rôle fondamental des processus physiques dans le contrôle de la disponibilité des ressources pour les producteurs primaires [50]. Dans les deux écosystèmes, l'intensification du mélange vertical pendant les marées de vives-eaux assure le renouvellement de la couche euphotique en silicates (pratiquement épuisés lors de la première floraison de diatomées). Si l'apport d'énergie auxiliaire engendrée par ces marées est utilisé immédiatement par le phytoplancton en Manche (faible turbidité des eaux), la turbidité plus importante des eaux de la rade fait que les cellules sont dans un état d'adaptation physiologique précaire qui ne leur permet pas de rentabiliser toute l'énergie dissipée dans l'écosystème pendant les viveseaux. L'accroissement de la production primaire n'a lieu qu'avec le ralentissement du mélange vertical, lors de la morte-eau suivante [50]. La réponse du microphytobenthos aux variations de l'intensité du mélange turbulent (et de la lumière) semble également dépendre de l'alternance vive-eau/morte-eau, dans la mesure où (1) les maxima de biomasse chlorophyllienne dans le sédiment ont été observés en morte-eau aux trois stations; (2) les biomasses minimales mesurées entre le 8 et le 12 juillet ont coïncidé avec une vive-eau (coefficient 87), et (3) l'augmentation progressive de la biomasse chlorophyllienne à RO lors des mortes-eaux successives de septembre s'est déroulée parallèlement à la baisse des coefficients de marée (coeff. 51-48 le 13 septembre, contre 31 le 29 septembre). Une évolution comparable en fonction des cycles de marée a été observée dans les vases intertidales de la ria du Dourduff [52].

L'absence de dénombrements cellulaires dans cette étude conduit à une incertitude majeure sur l'origine des pigments photosynthétiques mesurés dans le sédiment (phytoplancton sédimenté, microphytobenthos autochtone ou débris de macrophytes). Ainsi, même si le site profond de la Manche Ouest est colonisé par des microphytes benthiques (Cahoon et al. [8] indiquent la présence de microphytobenthos par $285 \mathrm{~m}$ en Caroline du Nord), l'intensité du mélange vertical y favorise l'apport de cellules phytoplanctoniques vivantes à l'interface eau-sédiment [50]. La comparaison de l'évolution spatio-temporelle des bio- 

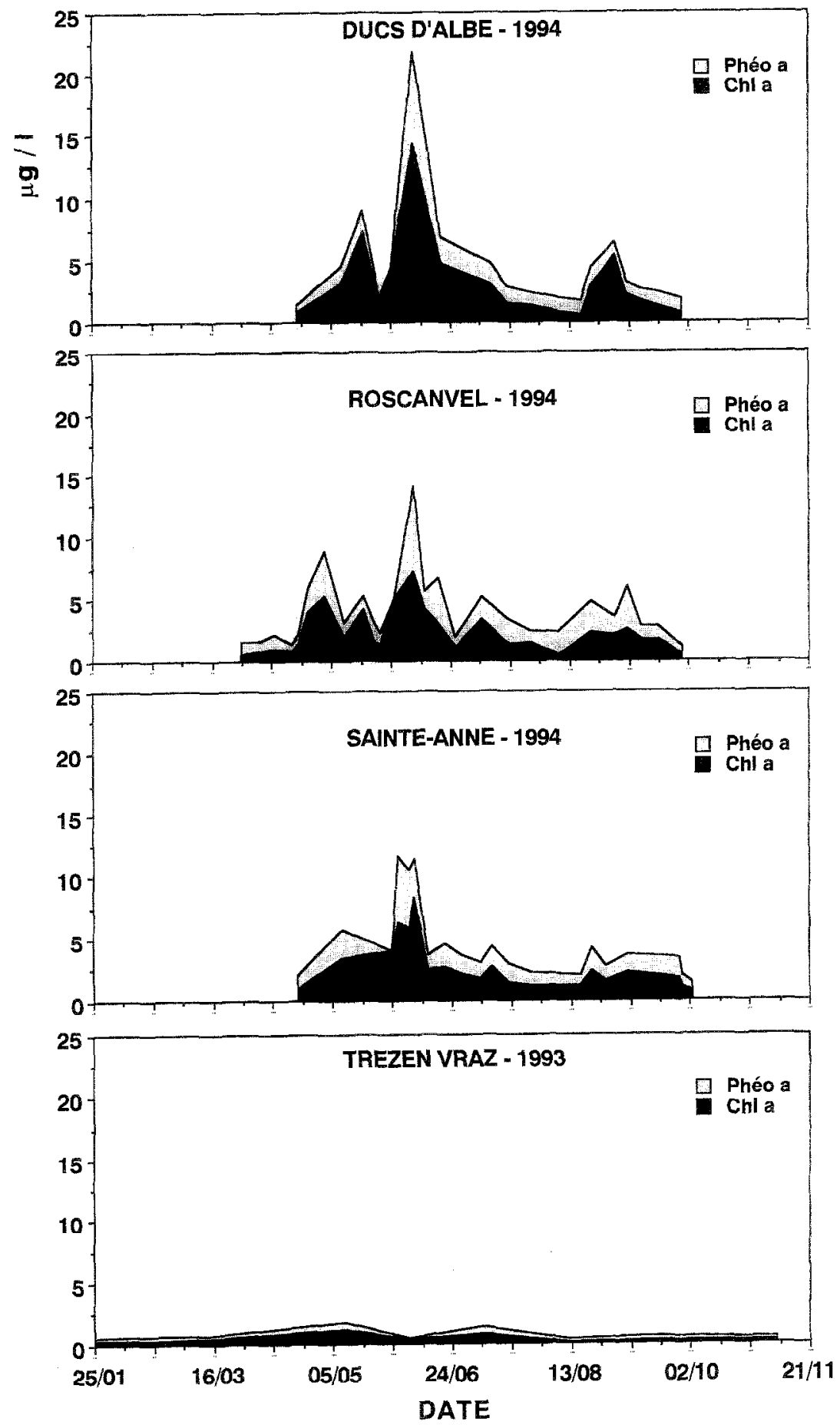

Figure 7. Concentrations en chlorophylle $a$ et en phéophytine $a$ dans la couche d'eau de fond $\left(\mu \mathrm{g} L^{-1}\right.$ ) en rade de Brest (1994; modifié d'aprè Chauvaud et al. [101) et en Manche occidentale (1993, Ragueneau, comm. pers.).

Figure 7. Bottom-water chlorophyll $a$ and phaeophytin $a$ concentrations ( $\mu \mathrm{L}^{-1}$ ) in the Bay of Brest (1994; modified from Chauvand et al [10]) and in the western English Channel (1993, Ragueneau, pers. comm.). 
masses pigmentaires pélagiques et benthiques avec celle des paramètres de l'environnement permet cependant d'identifier quelques processus déterministes en matière de couplage pélagos-benthos. Une caractéristique commune aux trois sites de la rade est le synchronisme des floraisons planctoniques et benthiques lorsqu'elles sont associées à une crue de l'Aulne ou de l'Elorn, alors qu'un décalage d'une à trois semaines est observé en l'absence de crue (l'origine de ces variations reste à déterminer).

$S A$ : l'augmentation des concentrations pigmentaires dans les sédiments de surface (0-15 mm) le 21 juin 1994 a lieu deux à trois semaines après la floraison planctonique $\left(8,3 \mu \mathrm{g} \mathrm{Chl} a \mathrm{~L}^{-1}\right.$ le 9 juin dans l'eau de fond contre $2,7 \mu \mathrm{g} \mathrm{L}^{-1}$ le 21 juin ; figure 7 d'après [10]). Le pic secondaire de Chl $a$ observé le 18 août dans la couche 0 $5 \mathrm{~mm}$ coïncide par contre avec un pic de Chl $a$ de faible amplitude dans la colonne d'eau $\left(2,3 \mu \mathrm{g}\right.$ Chl $a \mathrm{~L}^{-1}$ dans l'eau de fond ; figure 7). L'origine de cette floraison estivale est à rechercher dans une crue de l'Elorn une semaine avant (débit journalier moyen de $12 \mathrm{~m}^{3} \mathrm{~s}^{-1}$ [10]). Le pic automnal de phéo $a$ dans les sédiments superficiels est associé à des teneurs faibles en phéo $a$ de la colonne d'eau $\left(0,9 \mu \mathrm{g} \mathrm{L}^{-1}\right.$ dans l'cau de fond lc 29 scptcmbrc ; figure 7 ) pour laquelle le bloom planctonique automnal est enregistré le 6 septembre $\left(2,2 \mu \mathrm{g}\right.$ Chl $\left.a \mathrm{~L}^{-1}\right)$. Dans la mesure où les trois pics de phéo $a$ observés dans la couche $0-5 \mathrm{~mm}$ du sédiment en 1994 ont lieu une à trois semaines après trois pics de Chl $a$ dans la colonne d'eau, le rôle joué par la sédimentation des blooms phytoplanctoniques dans la dynamique pigmentaire au niveau du sédiment semble établi à cette station. Si l'augmentation cstivale des tencurs sédimentaires en phéo a reflète l'intensification de l'activité de broutage par les herbivores [53], elle doit aussi refléter le stockage du matériel détritique pendant l'été [47].

$D A$ : le pic de Chl $a$ du 19 mai dans le sédiment, associé à un bloom de phytoplancton $\left(7,4 \mu \mathrm{g}\right.$ Chl $a \mathrm{~L}^{-1}$ dans l'eau de fond ; figure 7), s'est installé en parallèle à l'augmentation du débit des rivières [10]. En particulier, le débit de l'Aulne a augmenté régulièrement à partir du 15 mai 1994 pour atteindre $65 \mathrm{~m}^{3} \mathrm{~s}^{-1}$ le 25 mai et $75 \mathrm{~m}^{3} \mathrm{~s}^{-1}$ le 31 mai. Les très fortes concentrations pigmentaires dans l'eau surnageante des carottes le 19 mai indiqueraient une sédimentation massive du "bloom" planctonique printanier à cette station et/ou une remise en suspension du sédiment. Comme à $\mathrm{SA}$, le pic de concentration en $\mathrm{Chl} a$ dans le sédiment le 21 juin est décalé de deux semaines par rapport à la principale floraison phytoplanctonique observée en 1994 (14,3 $\mu \mathrm{g} \mathrm{Chl} a \mathrm{~L}^{-1}$ le 9 juin dans l'eau de fond ; figure 7), mais le pic estival de microphytes (entre le 28 juillet et la mi-août) est associé à des concentrations pigmentaires minimales dans la colonne d'eau (figure 7) et à l'interface eau-sédiment.

Il semble donc que les floraisons benthiques à DA se développent indépendamment des floraisons planctoniques pendant l'été. L'absence de crues à cette période et les mortes-eaux entraîneraient une stabilisation du milieu favorable à la croissance des microphytes benthiques [11, $23,29,47,52]$, alors que les nutriments ne seraient pas limitants dans le sédiment. Les fortes teneurs du sédiment en phéopigments et les faibles valeurs du rapport $\mathrm{Chl} a /$ phéo $a$ illustreraient l'importance du broutage des microphytes et du stockage du matériel détritique sur ce site.

$R O$ : le pic printanier (19 mai) de Chl $a$ dans les sédiments de surface $(0-10 \mathrm{~mm})$ suit d'une quinzaine de jours la première floraison phytoplanctonique et coüncide avec la seconde $\left(5,3\right.$ et $4,1 \mu \mathrm{g} \mathrm{Chl} a \mathrm{~L}^{-1}$ dans l'eau de fond les 3 et 19 mai ; figure 7). Comme à DA, ce pic est corrélé aux augmentations de la température de l'eau et du débit des rivières. En ce qui concerne la colonne d'eau, les trois floraisons successives de mai-début juin (amplitudes moyennes, pas de bloon majeur) se sont déroulées pendant une crue, soulignant ainsi le rôle joué par les apports des bassins versants sur la dynamique des populations de microalgues à cette station [10]. Le pic automnal est précédé d'une à trois semaines par la dernière floraison phytoplanctonique $\left(2,6 \mu \mathrm{g} \mathrm{Chl} a \mathrm{~L}^{-1}\right.$ et $3,4 \mu \mathrm{g}$ phéo $a \mathrm{~L}^{-1}$ dans l'eau de fond le 6 septembre ; figure 7 ).

$T V$ : les variations temporelles de la biomasse pigmentaire dans les sédiments sont de faible amplitude, reflétant à la fois le caractère peu perturbé du site (mer ouverte), sa profondeur et les lacunes de l'échantillonnage. La périodicité et le mode d'échantillonnage (prélèvements mensuels ou bimestriels et utilisation d'un carottier ne permettant pas d'effectuer des coupes sédimentaires) ne permettent pas de conclure quant au couplage pelagos-benthos sur ce site.

\section{CONCLUSION}

L'un des objectifs de ce travail était la mise au point d'un protocole d'extraction des pigments chlorophylliens (Chl $a$ et phéo $a$ ) dans les sédiments côtiers des écosystèmes macrotidaux de la rade de Brest et de la Manche ouest. La comparaison de deux méthodes d'extraction a montré que 1'extraction instantanée en trois étapes et à température ambiante des pigments était la plus complète, 
confirmant ainsi les résultats de Barlow et al. [3]. L'analyse pigmentaire des sédiments étudiés montre l'apparition de floraisons microphytobenthiques fortement dépendantes, en rade de Brest, de l'évolution des facteurs physico-chimiques du milieu (crues des rivières, lumière, température, alternance vive-eau/morte-eau). Dans les deux écosystèmes, la dynamique spatio-temporelle des processus illustre l'importance des couplages physiquebiologie et pélagos-benthos pour la production primaire microphytobenthique, via les apports en sels nutritifs, la température de l'eau, le rayonnement solaire et l'intensité du mélange turbulent sur la verticale. La composition spécifique des cellules algales dans le sédiment et à l'interface, et la remise en suspension des microphytes mériteraient de faire l'objet d'une étude spécifique, afin de caractériser notamment le piégeage des pigmenis d'origine planctonique ou détritique dans le sédiment.

\section{Remerciements}

Les auteurs remercient Roger Kerouel et Philippe Cann (Centre Ifremer de Brest), Catherine Riaux-Gobin (Laboratoire d'océanographie biologique, URA 2071 CNRS), Olivier Ragueneau (Laboratoire Bioflux, UMR 6539 CNRS), Pierre Le Corre et Stéphane l'Helguen (Laboratoire d'océanographie chimique, UBO), et D. Byssus (LSRF) pour leurs conseils et leur aide dans la mise au point du protocole d'analyse et pour leurs remarques et suggestions dans la rédaction du manuscrit.

\section{RÉFÉRENCES}

[1] Abaychi J.K., Riley J.P., The determination of phytoplankton pigments by high-performance liquid chromatography, Anal. Chim. Acta 107 (1979) 1-11

[2] Aminot A., Chaussepied M., Manuel des analyses chimiques en milieu marin. Pub., CNEXO 15 (1983) 177-192.

[3] Barlow R.G., Collos Y., Maestrini S.Y., Roy S., Microphytobenthic pigments in a salt marsh pond determined by HPLC and spectrophotometry, Mar. Microb. Food Webs 4 (1990) $117-128$.

[4] Blanchard G.F., Cariou-Le Gall V., Photosynthetic characteristics of microphytobenthos in Marennes-Oléron Bay, France: Preliminary results, J. Exp. Mar. Biol. Ecol. 182 (1994) 1-14.

[5] Bodin P., Boucher D., Guillou J., Guillou M., The trophic system of the benthic communities in the Bay of Douarnenez (Brittany), in : Gibbs P.E., (éd.), Proc. 19th E.M.B.S., Plymouth, Devon (U.K.), 16-21 sept. 1984, Cambridge University Press, 1985, 361-370.

[6] Brown L.M., Hargrave B.T., Mackinnon M.D., Analysis of chlorophyll $a$ in sediments by high-pressure liquid chromatography, Can. J. Fish. Aquat. Sci. 38 (1981) 205-214.

[7] Cadée G.C., Hegeman J., Distribution of primary production of the benthic microflora and accumulation of organic matter on a tidal flat area, Balgzand, Dutch Wadden Sea. Neth. J. Sea Res. 11 (1977) 24-41.

[8] Cahoon L.B., Redman R.S., Tronzon C.R., Benthic microalgal biomass in sediments of Onslow Bay, North Carolina, Estuar. Coast. Shelf Sci. 57 (1990) 805-816.

[9] Chassé C., Głémarec M., Principes généraux de la classification des fonds pour la cartographie biosédimentaire, J. Rech. Océanogr. 1 (3) (1976) 1-11.
[10] Chauvaud L., Thouzeau G., Grall J., Experimental collection of great scallop postlarvae and other benthic species in the Bay of Brest: settlement patterns in relation to spatio-temporal variability of environmental factors, Aquaculture Int. 4 (1996) 263-288.

[11] Colijn F., Dijkema K.S., Species composition of benthic dia. toms and distribution of chlorophyll $a$ on an intertidal flat in the Dutch Wadden Sea, Mar. Ecol. Prog. Ser. 4 (1981) 9-21.

[12] Colijn F., De Jonge V.N., Primary production of microphytobenthos in the Ems-Dollard estuary, Mar. Ecol. Prog. Ser. 14 (1984) 185-196.

[13] Colocoloff M., Recherches sur la production primaire d'un fond sableux, 2. Biomasse et production, Tethys 4 (4) (1973) $779-800$.

[14] Daehnick A.E., Sullivan M.J., Moncrciff C.A., Primary production of the sand microflora in seagrass beds of Mississippi Sound, Bot. Mar. 35 (1992) 131-139.

[15] Daemen E.A.M.J., Comparison of methods for the determination of chlorophyll in estuarine sediments, Neth. J. Sea Res. 20 (1986) 21-28.

[16] De Jonge V.N., Fluctuations in the organic carbon to chlorophyll $a$ ratios for estuarine benthic diatom populations, Mar. Ecol. Prog. Ser. 2 (1980) 345-353.

[17] De La Giraudière I., Laborde P., Romano J.C., HPLC determination of chlorophylls and brcakdown products in surface microlayers, Mar. Chem. 26 (1989) 189-204.

[18] Del Amo Y., Le Pape O., Quéguiner B., Tréguer P., Ménesguen A., Cann P., Cycles biogéochimiques du carbone, de l'azote et du silicium. Succession d'espèces phytoplanctoniques, Actes de colloque, $3^{\text {es }}$ Rencontres scientifiques internationales, programme Rade, 14-16 mars 1995, Brest, I (1995) 59-79. 
[19] Delgado M., De Jonge V.N., Peletier H., Experiments on resuspension of natural microphytobenthos populations, Mar. Biol. 108 (1991) 321-328.

[20] Elliott J.M., Some methods for statistical analysis of samples of benthic invertebrates, Freshw. Biol. Ass. Sci. Pub. 25 (1977) $144 \mathrm{p}$.

[21] Falkowski P.G., Sucher J., Rapid quantitative separation of chlorophylls and their degradation products by high-performance liquid chromatography, J. Chromatogr. 213 (1981) 349-351.

[22] Hafsaoui M., Quéguiner B., Tréguer P., Production primaire et facteurs limitant la croissance du phytoplancton en Rade de Brest (1981 1983), Oceanis 11 (1985) 213222.

[23] Holland R.E., Zingmark R.G., Dean J.M., Quantitative evidence concerning the stabilization of sediments by marine benthic diatoms, Mar. Biol. 27 (1974) 191-196.

[24] Holm-Hansen O., Lorenzen C.J., Holmes R.W., Strickland J.D.H., Fluorometric determination of chlorophyll, J. Cons. Int. Explor. Mer 30 (1965) 3-15.

[25] Holm-Hansen O., Riemann B., Chlorophyll $a$ determination: improvements in methodology, Oikos 30 (1978) 438-447.

[26] Jean F., Modélisation à l'état stable des transferts de carbone dans le réseau trophique benthique de la Rade de Brest (France), Thèse Doc. Océanogr. Biol. Univ. Bretagne Occidentale, Brest (1994), 170 p.

[27] Jeffrey S.W., An improved thin-layer chromatographic technique for marine phytoplankton pigments, Limnol. Oceanogr. 26 (1981) 191-197.

[28] Jeffrey S.W., Humphrey G.F., New spectrophotometric equations for determining chlorophylls $a, b, c 1$ and $c 2$ in higher plants, algae, and natural phytoplankton, Biochem. Physiol. Pflanz. 167 (1975) 191-194.

[29] Jenness M.I., Duineveld G.C.A., Effects of tidal currents on chlorophyll a content of sandy sediments in the Southern North Sea, Mar. Ecol. Prog. Ser. 21 (1985) 283-287.

[30] Joint I.R., Microbial production of an estuarine mudflat, Estuar. Coast. Mar. Sci. 7 (1978) 185-195.

[31] Lehman P.W., Comparaison of chlorophyll $a$ and carotenoid pigments as predictors of phytoplankton biomass, Mar. Biol. 65 (1981) 237-244.

[32] Lenz J., Fritsche P., The estimation of chlorophyll $a$ in seawater samples: a comparative study on retention in glass-fibre and membrane filter and on the reliability of two storage methods, Arch. Hydrobiol. Beich. Ergeb. Limnol. 14 (1980) $46 \mathrm{p}$.

[33] Le Pape O., Del Amo Y., Cann P., Ménesguen A., Aminot A., Quéguiner B., Tréguer P., Resistance of a coastal ecosystem under increasing eutrophic conditions: the Bay of Brest (France), a semi-enclosed zone of western Europe, Cont. Shelf Res. 16 (15) (1996) 1885-1907.

[34] Loftus M.E., Carpenter J.H., A fluorimetric measurement for determining chlorophylls $a, b$ and $c$., J. Mar. Res. 29 (1971) 319-338.
[35] Lorenzen C.J., A method for the continuous measurement of in vivo chlorophyll concentration, Deep-Sea Res. 13 (1966) 223-227.

[36] Lorenzen C.J., Determination of chlorophyll and pheopigments: spectrophotometric equations, Limnol. Oceanogr. 12 (1967) 343-346.

[37] Lorenzen C.J., Chlorophyll $b$ in the eastem North Pacific Ocean, Deep-Sea Res. 28A (1981) 1049-1056.

[38] Lorenzen C.J., Downs J.N., The specific absorption coefficients of chlorophyllide $a$ and phaeophorbide $a$ in $90 \%$ acetone, and comments on the fluorometric determination of chlorophyll and pheopigments, Limnol. Oceanogr. 31 (1986) $449-452$.

[39] Lorenzen C.J., Jeffrey S.W., Determination of chlorophyll in seawater, UNESCO Technical Papers in Marine Science 35 (1980) $20 \mathrm{p}$.

[40] Mantoura R.F.C., Llewellyn C.A., The rapid determination of algal chloruphyll and carotenoid pigments and their breakdown products in natural waters by reverse-phase highperformance liquid chromatography, Anal. Chim. Acta 151 (1983) 297-314.

[41] Marker A.F.H., Some problems arising from the estimation of chlorophyll $a$ and phaeophytin $a$ in methanol, Limnol. Oceanogr. 22 (3) (1977) 578-579.

[42] Neveux J., Biomasse phytoplanctonique, in: Jacques G., (éd.), Phytoplancton : biomasse, production, numération et culture, Édition du castillet, Perpignan, 1979, 21-35.

[43] Parsons T.R., A new method for the microdetermination of chlorophyll $c$ in sea water, J. Mar. Res. 21 (1963) 164-171.

[44] Parsons T.R., Strickland J.D.H., Discussion of spectrophotometric determination of marine-plant pigments, with revised equations for ascertaining chlorophylls and carotenoids, J. Mar. Res. 21 (1963) 155-163.

[45] Patterson J., Parsons T.R., Distribution of chlorophyll $a$ and degradation products in various marine materials, Limnol. Oceanogr. 8 (1963) 355-356.

[46] Plante-Cuny M-R., Pigments photosynthétiques et production primaire des fonds meubles néritiques d'une région tropicale (Nosy-Bé, Madagascar), Trav. Doc. Orstom 96 (1978) 1-359.

[47] Plante R., Plante-Cuny M.R., Reys J.P., Photosynthetic pigments of sandy sediments on the North Mediterranean coast: their spatial distribution and its effect on sampling strategies, Mar. Ecol. Prog. Ser. 34 (1986). 133-141.

[48] Quéguiner B., Tréguer P., Freshwater outflow effects in a coastal macrotidal ecosystem as revealed by hydrological, chemical and biological variabilities (Bay of Brest, western Europe), in : S. Skreslet (éd.), The role of freshwater outflow in coastal marine ecosystems, NATO ASI Series G 7, 1986, 219-230.

[49] Ragueneau O., De Blas Varela E., Tréguer P., Quéguiner B., Del Amo Y., Phytoplancton dynamics in relation to the biogeochemical cycle of silicon in a coastal ecosystem of western Europe, Mar. Ecol. Prog. Ser. 106 (1994) 157-172.

[50] Ragueneau O., Quéguiner B., Tréguer P., Contrast in biological responses to tidally-induced vertical mixing for two macro- 
tidal ecosystems of western Europe, Estuar. Cuast. Shelf Sci. 42 (5) (1996) 645-665.

[51] Redden A.M., Thompson R.J., Deibel D., Effects of short and long term freezing of chloropigments in cultured diatoms and bivalve digestive gland and faeces as determined by standard fluorometry and HPLC, Arch. Hydrobiol. 129 (1993) 67-87.

[52] Riaux C., La chlorophylle $a$ dans un sédiment estuarien de Bretagne Nord, Ann. Inst. Océanogr. Paris, 58 (2) (1982) 185 203.

[53] Riaux-Gobin C., Llewellyn C.A., Klein B., Microphytobenthos from two subtidal sediments from North Brittany. II, Variations of pigment compositions and concentrations determined by HPLC and conventional techniques, Mar. Ecol. Prog. Ser. 40 (1987) 275-283.

[54] Riaux-Gobin C., Klein B., Microphytobenthic biomass measurement using HPLC and conventional pigment analysis, in : Kemp P.F., Sherr B.F., Sherr E.B., Cole J.J. (éd.), Handbook of methods in aquatic microbial ecology, Lewis Publishers, London, 42, 1993, 369-376.

[55] Riaux-Gobin C., Klein B., Wafar M.V.M., Production primaire microphylobenthique d'une slikke de nord Brelagne : statification verticale, J. Exp. Mar. Biol. Ecol. 169 (1993) 215-231.

[56] Richards F.A., Thompson T.G., The estimation and characterization of plankton populations by pigments analysis. II, A spectrophotometric method for the estimation of plankton pigments, J. Mar. Res. 11 (2) (1952) 156-172.

[57] Riemann B., Carotenoid interference in the spectrophometric determination of chlorophyll degradation products from natural populations of phytoplankton, Limnol. Oceanogr. 23 (1978) 1059-1066.

[58] Round F.E., Benthic marine diatoms, Oceanogr. Mar. Biol. Annu. Rev. 9 (1971) 83-139.

[59] Shuman F.R., Lorenzen C.J., Quantitative degradation of chlo rophyll by a marine herbivore, Limnol. Oceanogr. 20 (1975) $580-586$.
[60] Steele J.H., Baird I.E., Producion ecology of a sandy beach, Limnol. Oceanogr. 13 (1) (1968) 14-25.

[61] Strickland J.D.H., Parsons T.R., A practical handbook of seawater analysis, Bull. Fish. Res. Board. Can. 167 (1968) 184-206.

[62] Sundbäck K., Jönsson B., Microphytobenthic productivity and biomass in sublittoral sediments of a stratified bay, southeastern Kattegat, J, Exp. Mar. Biol. 122 (1988) 63-81.

[63] Taasen J.P., Høisæter T., The shallow-water soft-bottom in Lindaspollene, Western Norway. IV, Benthic marine diatoms, seasonal density fluctuations, Sarsia 66 (1981) 293 p.

[64] Tett P., Kelly M.G., Hornberger G.M., A method for the spectrophotometric measurement of chlorophyll $a$ and phaeophytin $a$ in benthic microalgae, Limnol. Oceanogr. 20 (5) (1975) 887-896.

[65] Tett P., Kelly M.G., Hornberger G.M., Estimation of chlorophyll $a$ and phaeophytin $a$ in methanol, Limnol. Oceanogr. 22 (3) (1977) 579-580.

[66] Trees C.C., Kennicutt M.C., Brooks J.M., Errors associated with the standard fluorimetric determination of chlorophylls and phaeopigments, Mar. Chem. 17 (1985) 1-12.

[67] Varela M., Penas E., Primary production of benthic microalgae in an intertidal sand flat of the Ria de Arosa, NW Spain, Mar. Ecol. Prog. Ser. 25 (1985) 111-119.

[68] Wattimena S., Thouzeau G., Variabilité saisonnière de la structure trophique et de la biodiversité du macrobenthos en Manche occidentale, J. Rech. Océanogr. 20 (3-4) (1995) 153-154.

[69] Wright S.W., Jeffrey S.W., Mantuura R.F.C., Llewellyn C.A., Bjornland T., Repeta D., Welschmeyer N., Improved HPLC method for the analysis of chlorophylls and carotenoids from marine phytoplankton, Mar. Ecol. Prog. Ser. 77 (1991) 183196.

[70] Yentsch C.S., Menzel D.W., $\Lambda$ method for the determination of phytoplankton chlorophyll and phaeophytin by fluorescence, Deep Sea Res. 10 (1963) 221-231. 